\title{
Analysis of two-point statistics of cosmic shear
}

\section{Estimators and covariances}

\author{
P. Schneider ${ }^{1,2}$, L. van Waerbeke ${ }^{3,4}$, M. Kilbinger ${ }^{1}$, and Y. Mellier ${ }^{3,5}$ \\ 1 Institut f. Astrophysik u. Extr. Forschung, Universität Bonn, Auf dem Hügel 71, 53121 Bonn, Germany \\ 2 Max-Planck-Institut f. Astrophysik, Postfach 1317, 85741 Garching, Germany \\ 3 Institute d'Astrophysique de Paris, 98bis boulevard Arago, 75014 Paris, France \\ 4 Canadian Institute for Theoretical Astrophysics, 60 St Georges Str., Toronto, M5S 3H8 Ontario, Canada \\ 5 Observatoire de Paris, DEMIRM/LERMA, 61 avenue de l'Observatoire, 75014 Paris, France
}

Received 13 June 2002 / Accepted 12 September 2002

\begin{abstract}
Recently, cosmic shear, the weak lensing effect by the inhomogeneous matter distribution in the Universe, has not only been detected by several groups, but the observational results have been used to derive constraints on cosmological parameters. For this purpose, several cosmic shear statistics have been employed. As shown recently, all second-order statistical measures can be expressed in terms of the two-point correlation functions of the shear, which thus represents the basic quantity; also, from a practical point-of-view, the two-point correlation functions are easiest to obtain from observational data which typically have complicated geometry. We derive in this paper expressions for the covariance matrix of the cosmic shear twopoint correlation functions which are readily applied to any survey geometry. Furthermore, we consider the more special case of a simple survey geometry which allows us to obtain approximations for the covariance matrix in terms of integrals which are readily evaluated numerically. These results are then used to study the covariance of the aperture mass dispersion which has been employed earlier in quantitative cosmic shear analyses. We show that the aperture mass dispersion, measured at two different angular scales, quickly decorrelates with the ratio of the scales. Inverting the relation between the shear two-point correlation functions and the power spectrum of the underlying projected matter distribution, we construct estimators for the power spectrum and for the band powers, and show that they yields accurate approximations; in particular, the correlation between band powers at different wave numbers is quite weak. The covariance matrix of the shear correlation function is then used to investigate the expected accuracy of cosmological parameter estimates from cosmic shear surveys. Depending on the use of prior information, e.g. from CMB measurements, cosmic shear can yield very accurate determinations of several cosmological parameters, in particular the normalization $\sigma_{8}$ of the power spectrum of the matter distribution, the matter density parameter $\Omega_{\mathrm{m}}$, and the shape parameter $\Gamma$.
\end{abstract}

Key words. dark matter - gravitational lensing - large-scale structure of the Universe

\section{Introduction}

Cosmic shear, the distortion of the images of distant galaxies by the tidal gravitational field of intervening matter inhomogeneities, offers a direct way of probing the statistical properties of the large-scale (dark) matter distribution in the Universe, without making any assumption on the relation between dark and luminous matter (e.g., Blandford et al. 1991; Miralda-Escude 1991; Kaiser 1992, 1998; Jain \& Seljak 1997; Bernardeau et al. 1997; Schneider et al. 1998, hereafter SvWJK; van Waerbeke et al. 1999; Bartelmann \& Schneider 1999; Jain et al. 2000; White \& Hu 2000; see Mellier 1999 and Bartelmann \& Schneider 2001 for recent reviews). The first detections of cosmic shear on wide-field imaging data (Bacon et al. 2000, 2002; Kaiser et al. 2000; van Waerbeke et al. 2000, 2001, 2002; Wittman et al. 2000; Maoli et al. 2001; Rhodes et al. 2001; Hämmerle et al. 2002; Hoekstra et al. 2002; Refregier et al. 2002) has demonstrated the feasibility of this new window of observational cosmology, and yielded already the first constraints on cosmological parameters, most noticibly the normalization $\sigma_{8}$ of the dark matter power spectrum, but also on the matter density parameter $\Omega_{\mathrm{m}}$ (van Waerbeke et al. 2002; Hoekstra et al. 2002).

Most analytical work on cosmic shear has been done on second-order statistical measures of the distortion field, such as the shear correlation functions, the shear variance in an apertures, or the aperture mass (see Sect. 2 for a definition of these

Send offprint requests to: $\mathrm{P}$. Schneider,

e-mail: peter@astro.uni-bonn.de 
quantitities). Although higher-order statistical measures, such as the skewness of the shear (Bernardeau et al. 1997), are likely to yield additional, if not even superior constraints on cosmological parameters, their theoretical predictions are more uncertain at present. Recently, an estimator for the skewness of the shear was developed (Bernardeau et al. 2002a), and applied to wide-field survey data (Bernardeau et al. 2002b), yielding a significant detection.

In this paper we consider second-order statistical measures only. All of them can be derived in terms of the correlation functions, as shown in, e.g., Crittenden et al. (2002, hereafter C02) and Schneider et al. (2002, hereafter SvWM), and since the measurement of the correlation functions is easier in practice than the other second-order statistics (e.g., gaps in the data are easily dealt with), we consider the correlation functions as the fundamental observables from a cosmic shear survey. In order to use them for determining cosmological parameters, it is important to know a practical and unbiased estimator for them, and to determine the covariance of this estimator. Two effects enter this covariance: a random part, which is due to the intrinsic ellipticity of the galaxies from which the shear is measured, together with measurement noise, and sampling (or cosmic) variance. The first of these effects is expected to dominate on small angular scales, whereas the second determines the covariance for large separations. The covariance will depend not only on the total survey area, but also on the survey geometry. As has been pointed out by Kaiser (1998), in order to decrease the sampling variance on large scales, it may be favourable to choose a survey geometry that samples those scales sparsely. In order to design an optimized survey geometry, the covariance as a function of survey geometry needs to be calculated.

Here, we calculate the covariance matrices for the shear correlation functions binned in angular separation. In Sect. 2, we introduce our notation and briefly summarize the second-order cosmic shear measures and their interrelations. Unbiased estimators of the two basic correlation functions are derived in Sect. 3, togther with the corresponding unbiased estimators of the aperture mass and the shear dispersion. The covariance matrices of these correlation function estimators are then derived in Sect. 4, expressed in terms of a set of galaxy positions. From these expressions, the covariances can be determined for an arbitrary survey geometry. In a forthcoming paper (Kilbinger et al., in preparation), we shall use the results of Sect. 4 to design an optimized geometry for a planned cosmic shear survey.

For the case of a filled survey geometry, the ensemble average of these covariances can be further analyzed; using a few approximations, we express in Sect. 5 the covariances for this case in terms of integrals. The corresponding expressions have been evaluated, for a particular cosmological model, and are illustrated in a set of figures. In Sect. 6 we derive the covariance for the aperture mass dispersion, which can be expressed simply in terms of the covariances of the correlation functions. The variance of the aperture mass dispersion, as well as the covariance, is then explicitly calculated for a survey with filled geometry, showing that indeed the aperture mass at two angular scales decorrelates quickly as the scale ratio decreases away from unity.

We then turn in Sect. 7 to a simple estimator of the power spectrum of the projected cosmic density field, which can be expresed in terms of the correlation functions. Since the correlation functions will be known only over a finite range in angular separation, the simple estimator we derive is biased. We show that, provided the angular range on which the correlation functions can be measured is as large as can be expected with the next generation of cosmic shear surveys, this bias is indeed very small over a large range of wave numbers. We derive the covariance of the power spectrum estimator and calculate it explicitly for the filled survey geometry case; the resulting error bars on the estimated power spectrum are quite a bit smaller than one might have expected, given the simplicity of the approach. In Sect. 8 we consider the accuracy with which the parameters of the cosmological model can be constrained, given a survey area. In fact, by fitting the correlation function directly to model predictions, even the currently available cosmic shear surveys can yield fairly accurate constraints on cosmological parameter. Finally, we summarize our results in Sect. 9.

In this paper we shall assume that the observable shear is due to the tidal gravitational field of the cosmological matter distribution only; in this case, the two shear components are not mutually independent. This is due to the fact that the gravitational field is a gradient field. In the language of some recent papers (e.g., C02; Pen et al. 2002; SvWM), we thus assume that the shear field is a pure $E$-mode field. $B$-modes (or the "curl component") can in principle be generated if the intrinsic orientation of the galaxies from which the shear is measured are correlated, e.g. due to tidal interactions of dark matter halos in which these galaxies are formed (Croft \& Metzler 2000; Pen et al. 2000; Heavens et al. 2001; Catelan et al. 2001; Mackey et al. 2002; Brown et al. 2002). Also, the clustering of source galaxies in redshift space generates a $B$-mode contribution which, however, turns out to be fairly small (SvWM). This restriction to $E$-modes only affects the interrelations between various two-point statistics. Inclusion of $B$-modes would not change the results of Sects. 3 through 5, and much of Sects. 6 and 7 will also be left unaffected in the presence of a $B$-mode contribution; we shall indicate this in due course.

\section{Second-order measures of cosmic shear}

We follow here the notation of Bartelmann \& Schneider (2001). The power spectrum of the projected density field is denoted by $P_{K}(\ell)$, where $\ell$ is the Fourier variable on the sky.

The shear correlation functions are defined by considering pairs of positions $\boldsymbol{\vartheta}$ and $\boldsymbol{\theta}+\boldsymbol{\vartheta}$, and defining the tangential and cross-component of the shear $\gamma=\gamma_{1}+\mathrm{i} \gamma_{2}$ at position $\vartheta$ for this pair as

$\gamma_{\mathrm{t}}=-\mathcal{R e}\left(\gamma \mathrm{e}^{-2 \mathrm{i} \varphi}\right) ; \quad \gamma_{\times}=-\mathcal{I} \mathrm{m}\left(\gamma \mathrm{e}^{-2 \mathrm{i} \varphi}\right)$, 
where $\varphi$ is the polar angle of the separation vector $\boldsymbol{\theta}$. Then we define the two shear correlation functions (e.g., Kaiser 1992)

$\xi_{ \pm}(\theta):=\left\langle\gamma_{\mathrm{t}} \gamma_{\mathrm{t}}\right\rangle \pm\left\langle\gamma_{\times} \gamma_{\times}\right\rangle=\frac{1}{2 \pi} \int_{0}^{\infty} \mathrm{d} \ell \ell P_{\kappa}(\ell) J_{0,4}(\ell \theta)$

where the Bessel function $J_{0}\left(J_{4}\right)$ corresponds to the "+" ("-") correlation function. The shear dispersion in a circle of radius $\theta$ is defined by considering circular apertures and ensemble-averaging over the square of the complex shear; its relation to the power spectrum reads (e.g., Kaiser 1992)

$\left\langle|\gamma|^{2}\right\rangle(\theta)=\frac{1}{2 \pi} \int_{0}^{\infty} \mathrm{d} \ell \ell P_{K}(\ell) \frac{4\left[J_{1}(\ell \theta)\right]^{2}}{(\ell \theta)^{2}}$.

Furthermore, the aperture mass $M_{\text {ap }}$ in an aperture of radius $\theta$ is defined as a weighted average over the tangential shear component (see, e.g. Schneider 1996; SvWJK), and its dispersion is related to the power spectrum by

$\left\langle M_{\mathrm{ap}}^{2}\right\rangle(\theta)=\frac{1}{2 \pi} \int_{0}^{\infty} \mathrm{d} \ell \ell P_{K}(\ell) \frac{576\left[J_{4}(\ell \theta)\right]^{2}}{(\ell \theta)^{4}}$

where the same weight function in the definition of $M_{\text {ap }}$ as in SvWM was assumed.

All these second-order statistics are thus linearly filtered versions of the power spectrum $P_{\kappa}$, where the filter functions are quite different between the various statistics. For the correlation function $\xi_{+}$, the filter function $J_{0}(\eta)$ is very broad, about constant for $\eta \ll 1$, and oscillating for large $\eta$, with an amplitude decreasing as $\eta^{-1 / 2}$. The filter function $J_{4}(\eta)$ for $\xi_{-}$has the same slow decrease, but behaves as $\eta^{4}$ for small $\eta$, and is therefore more localized than the one for $\xi_{+}$. The filter function for the shear dispersion, $\left[2 J_{1}(\eta) / \eta\right]^{2}$, is a low-pass filter, i.e. constant for $\eta \ll 1$, and then decreasing in amplitude as $\eta^{-3}$ for large $\eta$. Finally, the filter function for the aperture mass dispersion is $\left[24 J_{4}(\eta) / \eta^{2}\right]^{2}$ and thus behaves like $\eta^{4}$ for small $\eta$, and decreases oscillatory as $\eta^{-5}$ for $\eta \rightarrow \infty$. Hence, $\left\langle M_{\mathrm{ap}}^{2}\right\rangle$ yields the most local estimate of the underlying power spectrum of the projected mass. On the other hand, because it is so localized, it contains less power in its filter, so that the value of $\left\langle M_{\mathrm{ap}}^{2}\right\rangle$ is smaller than that of $\left\langle|\gamma|^{2}\right\rangle$ on the same filter scale $\theta$.

The various second-order statistics of the shear are related to each other; in particular, they can all be expressed in terms of the two-point correlation functions, as was shown in C02, Pen et al. (2002) and SvWM. We briefly summarize the results here.

Making use of the orthogonality of Bessel functions, the power spectrum can be expressed in terms of the correlation functions $\xi_{+}(\theta)$ and $\xi_{-}(\theta)$, by multiplying Eq. (2) by $\theta J_{0}(\ell \theta)$ and $\theta J_{4}(\ell \theta)$, respectively, and then integrating over $\theta$, to obtain

$P_{K}(\ell)=2 \pi \int_{0}^{\infty} \mathrm{d} \theta \theta \xi_{+}(\theta) J_{0}(\ell \theta)=2 \pi \int_{0}^{\infty} \mathrm{d} \theta \theta \xi_{-}(\theta) J_{4}(\ell \theta)$.

These equations express the power spectrum directly in terms of the observable correlation function; however, in order to evaluate $P_{K}(\ell)$ from them, one would need to know the correlation functions for all angles. In Sect. 7 below, we shall investigate how well the power spectrum can be determined from knowing the correlation function over a limited range of separations.

The two Eqs. (2) and (5) allow us to express $\xi_{+}$in terms of $\xi_{-}$, and reversely (see SvWM for a derivation),

$\xi_{-}(\theta)=\xi_{+}(\theta)+\int_{0}^{\theta} \mathrm{d} \vartheta \vartheta \xi_{+}(\vartheta)\left(\frac{4}{\theta^{2}}-\frac{12 \vartheta^{2}}{\theta^{4}}\right) ; \quad \xi_{+}(\theta)=\xi_{-}(\theta)+\int_{\theta}^{\infty} \mathrm{d} \vartheta \vartheta \xi_{-}(\vartheta)\left(\frac{4}{\vartheta^{2}}-\frac{12 \theta^{2}}{\vartheta^{4}}\right)$.

Hence one can obtain $\xi_{-}(\theta)$ from the correlation function $\xi_{+}(\vartheta)$ in the interval $0 \leq \vartheta \leq \theta$, and so this relation can be directly applied to observational data, with a minor extrapolation to small separations. The reverse relation, expressing $\xi_{+}$in terms of $\xi_{-}$, is less useful in practice, owing to the infinite range of integration.

Next we express the shear dispersion (3) in terms of the correlation function, by inserting (5) into (3); this yields (van Waerbeke 2000; SvWM)

$\left\langle|\gamma|^{2}\right\rangle(\theta)=\int_{0}^{2 \theta} \frac{\mathrm{d} \varphi \varphi}{\theta^{2}} \xi_{+}(\varphi) S_{+}\left(\frac{\varphi}{\theta}\right)=\int_{0}^{\infty} \frac{\mathrm{d} \varphi \varphi}{\theta^{2}} \xi_{-}(\varphi) S_{-}\left(\frac{\varphi}{\theta}\right)$,

where

$S_{+}(x)=4 \int_{0}^{\infty} \frac{\mathrm{d} t}{t} J_{0}(x t)\left[J_{1}(t)\right]^{2}=\frac{1}{\pi}\left[4 \arccos \left(\frac{x}{2}\right)-x \sqrt{4-x^{2}}\right] H(2-x)$,

$S_{-}(x)=4 \int_{0}^{\infty} \frac{\mathrm{d} t}{t} J_{4}(x t)\left[J_{1}(t)\right]^{2}=\frac{x \sqrt{4-x^{2}}\left(6-x^{2}\right)-8\left(3-x^{2}\right) \arcsin (x / 2)}{\pi x^{4}} H(2-x)+\frac{4\left(x^{2}-3\right)}{x^{4}} H(x-2)$,

and $H(x)$ is the Heaviside step function. The first form for $S_{ \pm}$is obtained directly from inserting (5) into (3), after some renaming of variables, and the details of evaluating the integrals over the triple product of Bessel functions are given in SvWM. The same 
is true for Eqs. (10) below. We see that the function $S_{+}(x)$ vanishes for $x>2$, so that the shear dispersion can be expressed as a finite-range integral over the correlation function $\xi_{+}$; this is not the case for $S_{-}$.

Similarly, one can express the aperture mass dispersion in terms of the correlation functions, by inserting (5) into (4):

$$
\left\langle M_{\mathrm{ap}}^{2}\right\rangle(\theta)=\int_{0}^{2 \theta} \frac{\mathrm{d} \varphi \varphi}{\theta^{2}} \xi_{+}(\varphi) T_{+}\left(\frac{\varphi}{\theta}\right)=\int_{0}^{2 \theta} \frac{\mathrm{d} \varphi \varphi}{\theta^{2}} \xi_{-}(\varphi) T_{-}\left(\frac{\varphi}{\theta}\right),
$$

where

$$
\begin{aligned}
T_{+}(x) & =576 \int_{0}^{\infty} \frac{\mathrm{d} t}{t^{3}} J_{0}(x t)\left[J_{4}(t)\right]^{2} \\
& =\left\{\frac{6\left(2-15 x^{2}\right)}{5}\left[1-\frac{2}{\pi} \arcsin \left(\frac{x}{2}\right)\right]+\frac{x \sqrt{4-x^{2}}}{100 \pi}\left(120+2320 x^{2}-754 x^{4}+132 x^{6}-9 x^{8}\right)\right\} H(2-x), \\
T_{-}(x) & =576 \int_{0}^{\infty} \frac{\mathrm{d} t}{t^{3}} J_{4}(x t)\left[J_{4}(t)\right]^{2}=\frac{192}{35 \pi} x^{3}\left(1-\frac{x^{2}}{4}\right)^{7 / 2} H(2-x) .
\end{aligned}
$$

Both of these functions vanish for $x>2$, so that $\left\langle M_{\mathrm{ap}}^{2}\right\rangle$ can be expressed by a finite integral over either $\xi_{ \pm}$. The functions $S_{ \pm}$and $T_{ \pm}$are plotted in SvWM.

The fact that we can express the shear dispersion and the aperture mass dispersion directly in terms of the correlation function over a finite interval is expected, given that the estimators of both statistics include products of ellipticities of pairs of objects separated by no more than the diameter of the aperture. However, what could not have been guessed a priori is that $\left\langle M_{\text {ap }}^{2}\right\rangle(\theta)$

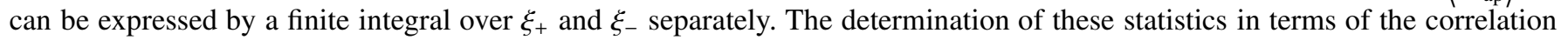
function is in practice easier than laying down apertures, owing to the holes and gaps in a data set; in addition, a comparison of the directly determined shear and aperture mass dispersion with those obtained from the correlation functions yields a useful check on the integrity of the data.

\section{Estimators}

We shall now consider practical estimators of the correlation functions and the other second-order statistics. The observable ellipticity $\epsilon_{i}$ of a galaxy image at angular position $\boldsymbol{\theta}_{i}$ is related to the intrinsic ellipticity $\epsilon_{i}^{\mathrm{s}}$ and the $\operatorname{shear} \gamma\left(\boldsymbol{\theta}_{i}\right)$ by

$\epsilon_{i}=\epsilon_{i}^{\mathrm{s}}+\gamma\left(\boldsymbol{\theta}_{i}\right)$

where it has been assumed that $|\gamma| \ll 1$ for this weak lensing relation to be valid. In addition to an ellipticity, each galaxy may carry a weight factor $w_{i}$ which reflects the precision with which its ellipticity can be determined - noisy objects can then be downweighted by assigning small values of $w_{i}$ to them (Hoekstra et al. 2000; Erben et al. 2001; Bacon et al. 2001 ; Pen et al. 2002). We shall assume that the correlation function is to be estimated in bins of angular width $\Delta \vartheta$, and define the function $\Delta_{\vartheta}(\phi)=1$ for $\vartheta-\Delta \vartheta / 2<\phi \leq \vartheta+\Delta \vartheta / 2$, and zero otherwise; hence, $\Delta_{\vartheta}(\phi)$ defines the bin at angle $\vartheta$. An estimator for the correlation function $\xi_{+}(\vartheta)$ is then

$\hat{\xi}_{+}(\vartheta)=\frac{\sum_{i j} w_{i} w_{j}\left(\epsilon_{i \mathrm{t}} \epsilon_{j \mathrm{t}}+\epsilon_{i \times} \epsilon_{j \times}\right) \Delta_{\vartheta}\left(\left|\boldsymbol{\theta}_{i}-\boldsymbol{\theta}_{j}\right|\right)}{N_{\mathrm{p}}(\vartheta)}, \quad N_{\mathrm{p}}(\vartheta)=\sum_{i j} w_{i} w_{j} \Delta_{\vartheta}\left(\left|\boldsymbol{\theta}_{i}-\boldsymbol{\theta}_{j}\right|\right) ;$

$N_{\mathrm{p}}(\vartheta)$ is the effective "number of pairs" in the bin considered (in fact, if all weights are unity, $N_{\mathrm{p}}$ is twice the number of pairs), and the tangential and cross components of the ellipticity are defined in analogy to the corresponding shear components in (1). The expectation value of this estimator is obtained by averaging over the source ellipticities, assumed to be randomly oriented, and an ensemble average of the shear field. Since

$\left\langle\epsilon_{i \mathrm{t}} \epsilon_{j \mathrm{t}}+\epsilon_{i \times} \epsilon_{j \times}\right\rangle=\sigma_{\epsilon}^{2} \delta_{i j}+\xi_{+}\left(\left|\boldsymbol{\theta}_{i}-\boldsymbol{\theta}_{j}\right|\right)$,

where $\sigma_{\epsilon}^{2}$ is the dispersion of the intrinsic galaxy ellipticity, we see immediately that $\hat{\xi}_{+}$is an unbiased estimator of $\xi_{+}$,

$\left\langle\hat{\xi}_{+}(\vartheta)\right\rangle=\xi_{+}(\vartheta)$

since the product $\delta_{i j} \Delta_{\vartheta}\left(\left|\boldsymbol{\theta}_{i}-\boldsymbol{\theta}_{j}\right|\right)$ vanishes for all pairs $i, j$. Analogously, an unbiased estimator for $\xi_{-}$is

$\hat{\xi}_{-}=\frac{\sum_{i j} w_{i} w_{j}\left(\epsilon_{i \mathrm{t}} \epsilon_{j \mathrm{t}}-\epsilon_{i \times} \epsilon_{j \times}\right) \Delta_{\vartheta}\left(\left|\boldsymbol{\theta}_{i}-\boldsymbol{\theta}_{j}\right|\right)}{N_{\mathrm{p}}(\vartheta)}, \quad\left\langle\hat{\xi}_{-}(\vartheta)\right\rangle=\xi_{-}(\vartheta)$. 
Next we obtain an unbiased estimator for the aperture mass dispersion. For that we assume that the centers of the bins on which the correlation function is calculated are described by $\vartheta_{i}=(i-1 / 2) \Delta \vartheta$, and that the aperture radius $\theta$ is an integer multiple of the bin width, $\theta=m \Delta \vartheta$. Then, the integrals in (9) are replaced by sums over the bins, yielding an estimator for $\left\langle M_{\mathrm{ap}}^{2}(\theta)\right\rangle$,

$\mathcal{M}(\theta)=\frac{\Delta \vartheta}{\theta^{2}} \sum_{i=1}^{2 m} \vartheta_{i}\left[K_{+} \hat{\xi}_{+}\left(\vartheta_{i}\right) T_{+}\left(\frac{\vartheta_{i}}{\theta}\right)+\left(1-K_{+}\right) \hat{\xi}_{-}\left(\vartheta_{i}\right) T_{-}\left(\frac{\vartheta_{i}}{\theta}\right)\right]$,

where $K_{+}$describes the relative contributions of the two expressions (9). For example, in the presence of $B$-modes, one needs to use $K_{+}=1 / 2(\mathrm{SvWM})$. Similarily, an unbiased estimator for the shear dispersion is

$\mathcal{S}(\theta)=\frac{\Delta \vartheta}{\theta^{2}}\left[K_{+} \sum_{i=1}^{2 m} \vartheta_{i} \hat{\xi}_{+}\left(\vartheta_{i}\right) S_{+}\left(\frac{\vartheta_{i}}{\theta}\right)+\left(1-K_{+}\right) \sum_{i=1}^{\infty} \vartheta_{i} \hat{\xi}_{-}\left(\vartheta_{i}\right) S_{-}\left(\frac{\vartheta_{i}}{\theta}\right)\right]$

again, in the presence of $B$-modes, $K_{+}=1 / 2$ shall be chosen (however, the infinite support of $S_{-}$requires the knowledge of the

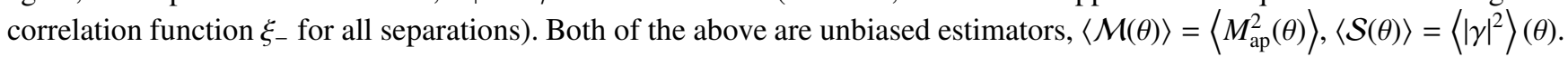

\section{Covariance of the estimators}

Next we calculate the covariance of the various estimators, starting with the correlation functions. Hence, we define

$\operatorname{Cov}\left(\hat{\xi}_{ \pm}, \vartheta_{1} ; \hat{\xi}_{ \pm}, \vartheta_{2}\right):=\left\langle\left(\hat{\xi}_{ \pm}\left(\vartheta_{1}\right)-\xi_{ \pm}\left(\vartheta_{1}\right)\right)\left(\hat{\xi}_{ \pm}\left(\vartheta_{2}\right)-\xi_{ \pm}\left(\vartheta_{2}\right)\right)\right\rangle$

Consider first the " ++ "-covariance function, for which one needs to evaluate

$\left\langle\hat{\xi}_{+}\left(\vartheta_{1}\right) \hat{\xi}_{+}\left(\vartheta_{2}\right)\right\rangle=\frac{1}{N_{\mathrm{p}}\left(\vartheta_{1}\right) N_{\mathrm{p}}\left(\vartheta_{2}\right)} \sum_{i j k l} w_{i} w_{j} w_{k} w_{l} \Delta_{\vartheta_{1}}(i j) \Delta_{\vartheta_{2}}(k l)\left\langle\left(\epsilon_{i 1} \epsilon_{j 1}+\epsilon_{i 2} \epsilon_{j 2}\right)\left(\epsilon_{k 1} \epsilon_{l 1}+\epsilon_{k 2} \epsilon_{l 2}\right)\right\rangle$,

where we defined $\Delta_{\vartheta}(i j) \equiv \Delta_{\vartheta}\left(\left|\boldsymbol{\theta}_{i}-\boldsymbol{\theta}_{j}\right|\right)$ and used the fact that $\epsilon_{i \mathrm{t}} \epsilon_{j \mathrm{t}}+\epsilon_{i \times} \epsilon_{j \times}=\epsilon_{i 1} \epsilon_{j 1}+\epsilon_{i 2} \epsilon_{j 2}$. Next, the four-point correlation function of the ellipticities needs to be evaluated. For that, we use (11) and expand the resulting expression. Only terms of even power in $\epsilon^{\mathrm{s}}$ and $\gamma$ survive the averaging over the source ellipticities and the ensemble average. Then,

$\left\langle\epsilon_{i \alpha} \epsilon_{j \beta} \epsilon_{k \mu} \epsilon_{l v}\right\rangle=\left\langle\gamma_{i \alpha} \gamma_{j \beta} \gamma_{k \mu} \gamma_{l v}\right\rangle+\frac{\sigma_{\epsilon}^{2}}{2}\left(\delta_{j l} \delta_{\beta v}\left\langle\gamma_{i \alpha} \gamma_{k \mu}\right\rangle+\delta_{j k} \delta_{\beta \mu}\left\langle\gamma_{i \alpha} \gamma_{l v}\right\rangle+\delta_{i l} \delta_{\alpha v}\left\langle\gamma_{j \beta} \gamma_{k \mu}\right\rangle+\delta_{i k} \delta_{\alpha \mu}\left\langle\gamma_{j \beta} \gamma_{l v}\right\rangle\right)+\left\langle\epsilon_{i \alpha}^{\mathrm{s}} \epsilon_{j \beta}^{\mathrm{s}} \epsilon_{k \mu}^{\mathrm{s}} \epsilon_{l v}^{\mathrm{s}}\right\rangle$,

valid for $i \neq j$ and $k \neq l$, as needed in (19); here, Greek letters are $\in\{1,2\}$. To evaluate the four-point function of the shear, we shall assume that the shear field is Gaussian, so that the four-point function can be written as a sum over products of two-point functions. We shall later comment on the effect this assuption has on the determination of the covariances. The four-point function of the intrinsic ellipticity also factorizes, since at most two of the indices $i, j, k, l$ are equal. Therefore,

$$
\begin{aligned}
\left\langle\epsilon_{i \alpha} \epsilon_{j \beta} \epsilon_{k \mu} \epsilon_{l v}\right\rangle= & \frac{\sigma_{\epsilon}^{2}}{2}\left(\delta_{j l} \delta_{\beta v}\left\langle\gamma_{i \alpha} \gamma_{k \mu}\right\rangle+\delta_{j k} \delta_{\beta \mu}\left\langle\gamma_{i \alpha} \gamma_{l v}\right\rangle+\delta_{i l} \delta_{\alpha v}\left\langle\gamma_{j \beta} \gamma_{k \mu}\right\rangle+\delta_{i k} \delta_{\alpha \mu}\left\langle\gamma_{j \beta} \gamma_{l v}\right\rangle\right) \\
& +\left\langle\gamma_{i \alpha} \gamma_{j \beta}\right\rangle\left\langle\gamma_{k \mu} \gamma_{l v}\right\rangle+\left\langle\gamma_{i \alpha} \gamma_{k \mu}\right\rangle\left\langle\gamma_{j \beta} \gamma_{l v}\right\rangle+\left\langle\gamma_{i \alpha} \gamma_{l v}\right\rangle\left\langle\gamma_{j \beta} \gamma_{k \mu}\right\rangle+\left(\frac{\sigma_{\epsilon}^{2}}{2}\right)^{2}\left(\delta_{i k} \delta_{j l} \delta_{\alpha \mu} \delta_{\beta v}+\delta_{i l} \delta_{j k} \delta_{\alpha \nu} \delta_{\beta \mu}\right) .
\end{aligned}
$$

The correlation functions of the shear components can be expressed as (Kaiser 1992)

$\left\langle\gamma_{i 1} \gamma_{j 1}\right\rangle=\frac{1}{2}\left[\xi_{+}(i j)+\xi_{-}(i j) \cos \left(4 \varphi_{i j}\right)\right] ;\left\langle\gamma_{i 2} \gamma_{j 2}\right\rangle=\frac{1}{2}\left[\xi_{+}(i j)-\xi_{-}(i j) \cos \left(4 \varphi_{i j}\right)\right] ;\left\langle\gamma_{i 1} \gamma_{j 2}\right\rangle=\frac{1}{2} \xi_{-}(i j) \sin \left(4 \varphi_{i j}\right)$,

where we have written $\xi_{ \pm}(i j) \equiv \xi_{ \pm}\left(\left|\boldsymbol{\theta}_{i}-\boldsymbol{\theta}_{j}\right|\right)$, and $\varphi_{i j}$ is the polar angle of the difference vector $\boldsymbol{\theta}_{i}-\boldsymbol{\theta}_{j}$. From these relations, one obtains for the covariance matrix

$$
\begin{aligned}
\operatorname{Cov}\left(\hat{\xi}_{+}, \vartheta_{1} ; \hat{\xi}_{+}, \vartheta_{2}\right)= & \frac{1}{N_{\mathrm{p}}\left(\vartheta_{1}\right) N_{\mathrm{p}}\left(\vartheta_{2}\right)}\left[\sigma_{\epsilon}^{4} \bar{\delta}\left(\vartheta_{1}-\vartheta_{2}\right) \sum_{i j} w_{i}^{2} w_{j}^{2} \Delta_{\vartheta_{1}}(i j)+2 \sigma_{\epsilon}^{2} \sum_{i j k} w_{i}^{2} w_{j} w_{k} \Delta_{\vartheta_{1}}(i j) \Delta_{\vartheta_{2}}(i k) \xi_{+}(j k)\right. \\
& \left.+\sum_{i j k l} w_{i} w_{j} w_{k} w_{l} \Delta_{\vartheta_{1}}(i j) \Delta_{\vartheta_{2}}(k l)\left(\xi_{+}(i l) \xi_{+}(j k)+\cos \left[4\left(\varphi_{i l}-\varphi_{j k}\right)\right] \xi_{-}(i l) \xi_{-}(j k)\right)\right]
\end{aligned}
$$

where the function $\bar{\delta}\left(\vartheta_{1}-\vartheta_{2}\right)$ is zero if the two separation bins are different, and is 1 if they are the same. The first term in (23) therefore contributes only to the diagonal terms in the covariance matrix. In the absence of shear correlations, the covariance matrix would be diagonal; correlation populates the off-diagonal elements of the covariance tensor. 


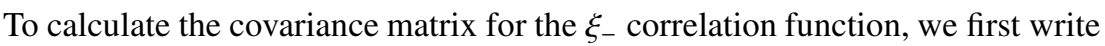

$\epsilon_{i \mathrm{t}} \epsilon_{j \mathrm{t}}-\epsilon_{i \times} \epsilon_{j \times}=\left(\epsilon_{i 1} \epsilon_{j 1}-\epsilon_{i 2} \epsilon_{j 2}\right) \cos 4 \varphi_{i j}+\left(\epsilon_{i 1} \epsilon_{j 2}+\epsilon_{i 2} \epsilon_{j 1}\right) \sin 4 \varphi_{i j}$

inserting this into the definition of the covariance matrix and performing the same step as for the " ++ " covariance, one finds

$$
\begin{aligned}
& \operatorname{Cov}\left(\hat{\xi}_{-}, \vartheta_{1} ; \hat{\xi}_{-}, \vartheta_{2}\right)=\frac{1}{N_{\mathrm{p}}\left(\vartheta_{1}\right) N_{\mathrm{p}}\left(\vartheta_{2}\right)}\left[\sigma_{\epsilon}^{4} \bar{\delta}\left(\vartheta_{1}-\vartheta_{2}\right) \sum_{i j} w_{i}^{2} w_{j}^{2} \Delta_{\vartheta_{1}}(i j)+2 \sigma_{\epsilon}^{2} \sum_{i j k} w_{i}^{2} w_{j} w_{k} \Delta_{\vartheta_{1}}(i j) \Delta_{\vartheta_{2}}(i k) \cos \left[4\left(\varphi_{i j}-\varphi_{i k}\right)\right] \xi_{+}(j k)\right. \\
& \left.\quad+\sum_{i j k l} w_{i} w_{j} w_{k} w_{l} \Delta_{\vartheta_{1}}(i j) \Delta_{\vartheta_{2}}(k l)\left(\cos \left[4\left(\varphi_{i j}-\varphi_{i l}-\varphi_{j k}+\varphi_{k l}\right)\right] \xi_{-}(i l) \xi_{-}(j k)+\cos \left[4\left(\varphi_{i j}-\varphi_{k l}\right)\right] \xi_{+}(i l) \xi_{+}(j k)\right)\right]
\end{aligned}
$$

Finally, the mixed covariance matrix can be calculated in the same manner, yielding

$$
\begin{aligned}
\operatorname{Cov}\left(\hat{\xi}_{+}, \vartheta_{1} ; \hat{\xi}_{-}, \vartheta_{2}\right)= & \frac{1}{N_{\mathrm{p}}\left(\vartheta_{1}\right) N_{\mathrm{p}}\left(\vartheta_{2}\right)}\left[2 \sigma_{\epsilon}^{2} \sum_{i j k} w_{i}^{2} w_{j} w_{k} \Delta_{\vartheta_{1}}(i j) \Delta_{\vartheta_{2}}(i k) \cos \left[4\left(\varphi_{i k}-\varphi_{j k}\right)\right] \xi_{-}(j k)\right. \\
& \left.+2 \sum_{i j k l} w_{i} w_{j} w_{k} w_{l} \Delta_{\vartheta_{1}}(i j) \Delta_{\vartheta_{2}}(k l) \cos \left[4\left(\varphi_{i l}-\varphi_{k l}\right)\right] \xi_{-}(i l) \xi_{+}(j k)\right]
\end{aligned}
$$

\section{Averaging over an ensemble of galaxy positions}

Given a model for the shear correlation, the covariances (23)-(25) can be calculated using the actual galaxy positions and their weight factors; in principle, this procedure is straightforward to apply to a given data set. Alternatively, given the geometry of a data field, then by randomly distributing galaxy positions the expected covariance of the shear correlation can be determined; this procedure can be used to design and optimize cosmic shear surveys (Kilbinger et al., in preparation). These calculations are, however, time-consuming, given the sum over three and four galaxy positions. It is therefore of interest to consider a relatively simple situation where fairly explicit expressions for the covariance matrices can be obtained. In particular, we shall calculate the ensemble average of the covariance matrices. We consider here a survey geometry which consists of a single data field of solid angle $A$ and galaxy number density $n$, so that $N=n A$ is the total number of galaxies in the survey. The survey geometry is assumed to be "simple", i.e. consisting of a simply connected region, say, a quadratic field. We shall assume that all weight factors are unity, $w_{i}=1$. Furthermore, we shall consider separations $\vartheta_{i}$ which are small compared to the "diameter" of the survey field, $\vartheta_{i}^{2} \ll A$; in this case, we can neglect "boundary effects" which otherwise would complicate the analysis tremendously. With these assumptions, the number of pairs in the bin characterized by $\vartheta$ is then

$N_{\mathrm{p}}(\vartheta)=A n 2 \pi \vartheta \Delta \vartheta n$

The ensemble average over galaxy positions is carried out by the averaging operator

$E=\prod_{i=1}^{N}\left(\frac{1}{A} \int_{A} \mathrm{~d}^{2} \theta_{i}\right)$

i.e. the galaxies are assumed to be randomly placed on the field. The average of the first term in (23) and (24) is simple and reads

$E\left(\frac{\sigma_{\epsilon}^{4} \bar{\delta}\left(\vartheta_{1}-\vartheta_{2}\right)}{N_{\mathrm{p}}\left(\vartheta_{1}\right) N_{\mathrm{p}}\left(\vartheta_{2}\right)} \sum_{i j} \Delta_{\vartheta_{1}}(i j)\right)=\frac{\sigma_{\epsilon}^{4}}{N_{\mathrm{p}}\left(\vartheta_{1}\right)} \bar{\delta}\left(\vartheta_{1}-\vartheta_{2}\right)=: D \bar{\delta}\left(\vartheta_{1}-\vartheta_{2}\right)$

As expected, this term depends explicitly on the bin size chosen, since $N_{\mathrm{p}} \propto \Delta \vartheta$. In practical units,

$D=3.979 \times 10^{-9}\left(\frac{\sigma_{\epsilon}}{0.3}\right)^{4}\left(\frac{A}{1 \mathrm{deg}^{2}}\right)^{-1}\left(\frac{n}{30 \operatorname{arcmin}^{-2}}\right)^{-2}\left(\frac{\vartheta}{1 \operatorname{arcmin}}\right)^{-2}\left(\frac{\Delta \vartheta / \vartheta}{0.1}\right)^{-1}$

As we shall see, the other terms are independent of the choice of the bins. To evaluate the other terms, one notes that the expectation value of all terms involving a sum over three galaxy positions can be written in terms of

$E_{\mathrm{abc}} \equiv E\left(\sum_{i j k} \Delta_{\vartheta_{1}}(i j) \Delta_{\vartheta_{2}}(i k) F_{\mathrm{a}}\left(\varphi_{i j}\right) F_{\mathrm{b}}\left(\varphi_{i k}\right) F_{\mathrm{c}}\left(\boldsymbol{\theta}_{k}-\boldsymbol{\theta}_{j}\right)\right)$, 
or linear combinations thereof. Applying the averaging operator, we note that there are $N(N-1)(N-2) \approx N^{3}$ permutations of galaxies, so that

$$
\begin{aligned}
E_{\mathrm{abc}} & =\frac{N^{3}}{A^{3}} \int \mathrm{d}^{2} \theta_{1} \int \mathrm{d}^{2} \theta_{2} \int \mathrm{d}^{2} \theta_{3} \Delta_{\vartheta_{1}}(12) \Delta_{\vartheta_{2}}(13) F_{\mathrm{a}}\left(\varphi_{12}\right) F_{\mathrm{b}}\left(\varphi_{13}\right) F_{\mathrm{c}}\left(\boldsymbol{\theta}_{3}-\boldsymbol{\theta}_{2}\right) \\
& =\frac{N^{3}}{A^{2}} \int_{\vartheta_{1}-\Delta \vartheta / 2}^{\vartheta_{1}+\Delta \vartheta / 2} \mathrm{~d} \phi_{1} \phi_{1} \int_{0}^{2 \pi} \mathrm{d} \varphi_{1} F_{\mathrm{a}}\left(\varphi_{1}\right) \int_{\vartheta_{2}-\Delta \vartheta / 2}^{\vartheta_{2}+\Delta \vartheta / 2} \mathrm{~d} \phi_{2} \phi_{2} \int_{0}^{2 \pi} \mathrm{d} \varphi_{2} F_{\mathrm{b}}\left(\varphi_{2}\right) F_{\mathrm{c}}\left(\phi_{2}-\phi_{1}\right),
\end{aligned}
$$

where in the second step we have written $\boldsymbol{\theta}_{2}=\boldsymbol{\theta}_{1}+\phi_{1}, \boldsymbol{\theta}_{3}=\boldsymbol{\theta}_{1}+\phi_{2}$, so that $\boldsymbol{\theta}_{3}-\boldsymbol{\theta}_{2}=\phi_{2}-\phi_{1}, \varphi_{1}$ and $\varphi_{2}$ denote the polar angles of $\phi_{1}, \phi_{2}$, respectively. After this substitution, the integral becomes independent of $\boldsymbol{\theta}_{1}$, which can be integrated to yield a factor $A$. Assuming that the bin width $\Delta \vartheta$ is small, one can evaluate the $\phi_{1}$ and $\phi_{2}$ integrals, to obtain

$\frac{E_{\mathrm{abc}}}{N_{\mathrm{p}}\left(\vartheta_{1}\right) N_{\mathrm{p}}\left(\vartheta_{2}\right)}=\frac{1}{(2 \pi)^{2} A n} \int_{0}^{2 \pi} \mathrm{d} \varphi_{1} F_{\mathrm{a}}\left(\varphi_{1}\right) \int_{0}^{2 \pi} \mathrm{d} \varphi_{2} F_{\mathrm{b}}\left(\varphi_{2}\right) F_{\mathrm{c}}\left(\begin{array}{c}\vartheta_{2} \cos \varphi_{2}-\vartheta_{1} \cos \varphi_{1} \\ \vartheta_{2} \sin \varphi_{2}-\vartheta_{1} \sin \varphi_{1}\end{array}\right)$.

Similarily, the expectation value of all terms involving a sum over four galaxy positions can be written in terms of

$$
\begin{aligned}
E_{\text {abcd }} & \equiv E\left(\sum_{i j k l} \Delta_{\vartheta_{1}}(i j) \Delta_{\vartheta_{2}}(k l) F_{\mathrm{a}}\left(\boldsymbol{\theta}_{k}-\boldsymbol{\theta}_{j}\right) F_{\mathrm{b}}\left(\boldsymbol{\theta}_{l}-\boldsymbol{\theta}_{i}\right) F_{\mathrm{c}}\left(\varphi_{i j}\right) F_{\mathrm{d}}\left(\varphi_{k l}\right)\right) \\
& =\frac{N^{4}}{A^{4}} \int \mathrm{d}^{2} \theta_{1} \int \mathrm{d}^{2} \theta_{2} \Delta_{\vartheta_{1}}(12) F_{\mathrm{c}}\left(\varphi_{12}\right) \int \mathrm{d}^{2} \theta_{3} \int \mathrm{d}^{2} \theta_{4} \Delta_{\vartheta_{2}}(34) F_{\mathrm{d}}\left(\varphi_{34}\right) F_{\mathrm{a}}\left(\boldsymbol{\theta}_{3}-\boldsymbol{\theta}_{2}\right) F_{\mathrm{b}}\left(\boldsymbol{\theta}_{4}-\boldsymbol{\theta}_{1}\right) \\
& =\frac{N^{4}}{A^{3}} \int \mathrm{d}^{2} \phi \int_{\vartheta_{1}-\Delta \vartheta / 2}^{\vartheta_{1}+\Delta \vartheta / 2} \mathrm{~d} \phi_{1} \phi_{1} \int_{0}^{2 \pi} \mathrm{d} \varphi_{1} F_{\mathrm{c}}\left(\varphi_{1}\right) \int_{\vartheta_{2}-\Delta \vartheta / 2}^{\vartheta_{2}+\Delta \vartheta / 2} \mathrm{~d} \phi_{2} \phi_{2} \int_{0}^{2 \pi} \mathrm{d} \varphi_{2} F_{\mathrm{d}}\left(\varphi_{2}\right) F_{\mathrm{a}}\left(\phi-\phi_{1}\right) F_{\mathrm{b}}\left(\phi+\phi_{2}\right),
\end{aligned}
$$

where in the second step we defined $\boldsymbol{\theta}_{2}=\boldsymbol{\theta}_{1}+\phi_{1}, \boldsymbol{\theta}_{4}=\boldsymbol{\theta}_{3}+\phi_{2}$; then, the arguments of the functions $F_{\mathrm{a}}$ and $F_{\mathrm{b}}$ become $\theta_{3}-\theta_{2}=\theta_{3}-\theta_{1}-\phi_{1}$ and $\theta_{4}-\theta_{1}=\theta_{3}-\theta_{1}+\phi_{2}$, i.e. they depend only on the difference $\phi=\theta_{3}-\theta_{1}$, so that the integral over $\theta_{3}$ can be carried out, yielding a factor $A$. Performing the $\phi_{1}$ and $\phi_{2}$ integration, assuming small bin width, one obtains

$\frac{E_{\mathrm{abcd}}}{N_{\mathrm{p}}\left(\vartheta_{1}\right) N_{\mathrm{p}}\left(\vartheta_{2}\right)}=\frac{1}{(2 \pi)^{2} A} \int_{0}^{\infty} \mathrm{d} \phi \phi \int_{0}^{2 \pi} \mathrm{d} \varphi_{1} F_{\mathrm{c}}\left(\varphi_{1}\right) \int_{0}^{2 \pi} \mathrm{d} \varphi_{2} F_{\mathrm{d}}\left(\varphi_{2}\right) \int_{0}^{2 \pi} \mathrm{d} \varphi F_{\mathrm{a}}\left(\psi_{\mathrm{a}}\right) F_{\mathrm{b}}\left(\psi_{\mathrm{b}}\right)$,

where the vectors

$\psi_{\mathrm{a}}=\left(\begin{array}{c}\phi \cos \varphi-\vartheta_{1} \cos \varphi_{1} \\ \phi \sin \varphi-\vartheta_{1} \sin \varphi_{1}\end{array}\right) ; \quad \psi_{\mathrm{b}}=\left(\begin{array}{c}\phi \cos \varphi+\vartheta_{2} \cos \varphi_{2} \\ \phi \sin \varphi+\vartheta_{2} \sin \varphi_{2}\end{array}\right)$

have been defined for later convenience.

Next we shall evaluate the second term of (23) which is of the form (28), with $F_{\mathrm{a}}=1=F_{\mathrm{b}}, F_{\mathrm{c}}(\psi)=\xi_{+}(|\psi|)$; inserting these expressions into (29), the expectation value of the second term in (23) becomes

$q_{++}=\frac{2 \sigma_{\epsilon}^{2}}{\pi A n} \int_{0}^{\pi} \mathrm{d} \varphi \xi_{+}\left(\sqrt{\vartheta_{1}^{2}+\vartheta_{2}^{2}-2 \vartheta_{1} \vartheta_{2} \cos \varphi}\right)$.

The expectation value of the third term in (23) can be calculated by splitting it up into three parts: in the first, $F_{\mathrm{a}}(\psi)=\xi_{+}(|\psi|)=$ $F_{\mathrm{b}}(\psi)$, and the other two are obtained by expanding the cosine-term, so that for one of them, $F_{\mathrm{a}}(\psi)=\cos 4 \varphi_{\psi} \xi_{-}(|\psi|)=F_{\mathrm{b}}(\psi)$, and for the other, cos is replaced by sin; here, $\varphi_{\psi}$ is the polar angle of $\psi$. Note that all three terms have $F_{\mathrm{c}}=1=F_{\mathrm{d}}$. The final result then reads

$E\left(\operatorname{Cov}\left(\hat{\xi}_{+}, \vartheta_{1} ; \hat{\xi}_{+}, \vartheta_{2}\right)\right)=D \bar{\delta}\left(\vartheta_{1}-\vartheta_{2}\right)+q_{++}+r_{+0}+r_{+1}$,

where $D$ and $q_{++}$have been defined in (27) and (32), respectively,

$r_{+0}=\frac{2}{\pi A} \int_{0}^{\infty} \mathrm{d} \phi \phi \int_{0}^{\pi} \mathrm{d} \varphi_{1} \xi_{+}\left(\left|\boldsymbol{\psi}_{\mathrm{a}}\right|\right) \int_{0}^{\pi} \mathrm{d} \varphi_{2} \xi_{+}\left(\left|\boldsymbol{\psi}_{\mathrm{b}}\right|\right)$,

$r_{+1}=\frac{1}{(2 \pi) A} \int_{0}^{\infty} \mathrm{d} \phi \phi \int_{0}^{2 \pi} \mathrm{d} \varphi_{1} \xi_{-}\left(\left|\psi_{\mathrm{a}}\right|\right) \int_{0}^{2 \pi} \mathrm{d} \varphi_{2} \xi_{-}\left(\left|\psi_{\mathrm{b}}\right|\right)\left[\cos 4 \varphi_{\mathrm{a}} \cos 4 \varphi_{\mathrm{b}}+\sin 4 \varphi_{\mathrm{a}} \sin 4 \varphi_{\mathrm{b}}\right]$,

and $\varphi_{\mathrm{a}}, \varphi_{\mathrm{b}}$ are the polar angles of $\boldsymbol{\psi}_{\mathrm{a}}, \boldsymbol{\psi}_{\mathrm{b}}$, respectively, $\cos 4 \varphi_{\mathrm{a}}=1-8 \psi_{\mathrm{a} 1}^{2} \psi_{\mathrm{a} 2}^{2} /\left|\psi_{\mathrm{a}}\right|^{4}, \sin 4 \varphi_{\mathrm{a}}=4 \psi_{\mathrm{a} 1} \psi_{\mathrm{a} 2}\left(\psi_{\mathrm{a} 1}^{2}-\psi_{\mathrm{a} 2}^{2}\right) /\left|\psi_{\mathrm{a}}\right|^{4}$, and the analogous expressions for $\varphi_{\mathrm{b}}$. Note that the $\varphi$-integration present in (30) has dropped out as the integrand depends only on $\varphi_{1}-\varphi$, and $\varphi_{2}-\varphi$; hence, the $\varphi$-integration can be carried out and one can use $\varphi=0$ in (31).

Several issues are worth mentioning: (1) only the first term containing the "delta function" depends on the bin width $\Delta \vartheta$; thus, the bin width only affects the autovariance. (2) All terms are proportional to $A^{-1}$; hence, the relative contribution of the terms 
is independent of the survey area, at least for separations well below the "diameter" of the survey area for which the foregoing procedure of the ensemble averaging is valid. (3) The terms denoted by " $r$ " are independent of the intrinsic ellipticity dispersion and of the number density of galaxies. Hence, these terms describe the cosmic variance and thus provide a limit on the accuracy of the determination of the correlation function for a given survey geometry, independent of the observing conditions which determine $n$.

The expectation values of the other covariance matrices are calculated in a similar manner. Consider the "--" covariance (24) next; the first term agrees with that of (23). For the second term, we can apply (29), after expanding the cosine; then $F_{\mathrm{a}}(\varphi)$ and $F_{\mathrm{b}}(\varphi)$ are either $\cos 4 \varphi$ or $\sin 4 \varphi$, and $F_{\mathrm{c}}(\psi)=\xi_{+}(|\psi|)$. Similarily, (30) can be applied to the third term of (24), after expanding the cosine; using (30) term by term, and combining the results afterwards, one obtains

$E\left(\operatorname{Cov}\left(\hat{\xi}_{-}, \vartheta_{1} ; \hat{\xi}_{-}, \vartheta_{2}\right)\right)=D \bar{\delta}\left(\vartheta_{1}-\vartheta_{2}\right)+q_{--}+r_{-0}+r_{-1}$,

where

$$
\begin{aligned}
q_{--} & =\frac{2 \sigma_{\epsilon}^{2}}{\pi A n} \int_{0}^{\pi} \mathrm{d} \varphi \xi_{+}\left(\sqrt{\vartheta_{1}^{2}+\vartheta_{2}^{2}-2 \vartheta_{1} \vartheta_{2} \cos \varphi}\right) \cos (4 \varphi), \\
r_{-0} & =\frac{1}{(2 \pi) A} \int_{0}^{\infty} \mathrm{d} \phi \phi \int_{0}^{2 \pi} \mathrm{d} \varphi_{1} \xi_{-}\left(\left|\psi_{\mathrm{a}}\right|\right) \int_{0}^{2 \pi} \mathrm{d} \varphi_{2} \xi_{-}\left(\left|\psi_{\mathrm{b}}\right|\right) \cos \left[4\left(\varphi_{1}+\varphi_{2}-\varphi_{\mathrm{a}}-\varphi_{\mathrm{b}}\right)\right] \\
r_{-1} & =\frac{1}{(2 \pi) A} \int_{0}^{\infty} \mathrm{d} \phi \phi \int_{0}^{2 \pi} \mathrm{d} \varphi_{1} \xi_{+}\left(\left|\psi_{\mathrm{a}}\right|\right) \int_{0}^{2 \pi} \mathrm{d} \varphi_{2} \xi_{+}\left(\left|\psi_{\mathrm{b}}\right|\right) \cos \left[4\left(\varphi_{1}-\varphi_{2}\right)\right] .
\end{aligned}
$$

Finally, the expectation value of (25), the mixed covariance, is calculated in a similar manner, yielding

$E\left(\operatorname{Cov}\left(\hat{\xi}_{+}, \vartheta_{1} ; \hat{\xi}_{-}, \vartheta_{2}\right)\right)=q_{+-}+r_{+-}$

with

$$
\begin{aligned}
q_{+-} & =\frac{2 \sigma_{\epsilon}^{2}}{\pi A n} \int_{0}^{\pi} \mathrm{d} \varphi\left[\sum_{k=0}^{4}\left(\begin{array}{l}
4 \\
k
\end{array}\right)(-1)^{k} \vartheta_{1}^{k} \vartheta_{2}^{4-k} \cos (k \varphi)\right]\left(\vartheta_{1}^{2}+\vartheta_{2}^{2}-2 \vartheta_{1} \vartheta_{2} \cos \varphi\right)^{-2} \xi_{-}\left(\sqrt{\vartheta_{1}^{2}+\vartheta_{2}^{2}-2 \vartheta_{1} \vartheta_{2} \cos \varphi}\right) \\
r_{+-} & =\frac{1}{\pi A} \int_{0}^{\infty} \mathrm{d} \phi \phi \int_{0}^{2 \pi} \mathrm{d} \varphi_{1} \xi_{+}\left(\left|\psi_{\mathrm{a}}\right|\right) \int_{0}^{2 \pi} \mathrm{d} \varphi_{2} \xi_{-}\left(\left|\boldsymbol{\psi}_{\mathrm{b}}\right|\right) \cos \left[4\left(\varphi_{2}-\varphi_{\mathrm{b}}\right)\right] .
\end{aligned}
$$

Hence, as already seen from (25), the cross-covariance matrix has no pure noise term from the intrinsic galaxy ellipticity dispersion as the other two covariance matrices.

We have obtained numerical estimates for the ensemble-averaged covariance matrices derived above (see Fig. 1). In the numerical estimates given in this paper (except for Sect. 8.2), we have used a standard set of parameters. The cosmological parameters are those of a by-now standard $\Lambda$-dominated universe, $\Omega_{\mathrm{m}}=0.3, \Omega_{\Lambda}=0.7$. The power spectrum of the density fluctuations is described by its primordial slope of $n=1$, a shape parameter $\Gamma=0.21$, and a normalization of $\sigma_{8}=1$. We used the fit formula of Bardeen et al. (1986) for the linear power spectrum, and the prescription of Hamilton et al. (1991) in the form given in Peacock \& Dodds (1996) to describe the non-linear evolution of the power spectrum ${ }^{1}$. Furthermore, we fix the survey properties to be described by a fiducial area of $A=1 \mathrm{deg}^{2}$, a number density $n=30 \mathrm{arcmin}^{-2}$ of source galaxies, and an intrinsic ellipticity dispersion of $\sigma_{\epsilon}=0.3$. The source galaxies were assumed to have a redshift distribution $p(z) \propto z^{2} \exp \left[-\left(z / z_{0}\right)^{1.5}\right]$, so that the mean redshift is $\bar{z}=1.505 z_{0}$. For the examples shown in Sects. 5 through 7 , we take $z_{0}=1$. Note that all covariances simply scale with $A^{-1}$, so that the results displayed here are easily translated to other survey sizes. This scaling is also implicitly implied when considering scales of order a degree or more - all the numerical estimates are for the ensemble averaged covariances, and their validity as given here depends on the assumption $\theta^{2} \ll A$.

Figure 2 displays in the left panel the square root of the (auto)variance of $\hat{\xi}_{+}(\vartheta)$ and $\hat{\xi}_{-}(\vartheta)$, with $\operatorname{Var}\left(\hat{\xi}_{ \pm} ; \vartheta\right)=\operatorname{Cov}\left(\hat{\xi}_{ \pm}, \vartheta ; \hat{\xi}_{ \pm}, \vartheta\right)$. To calculate the value of $D$ (27) which enters the diagonal part of the covariance matrix, we have assumed a bin width of $\Delta \vartheta=0.1 \vartheta$. The square root of the variance - or noise per bin - for $\xi_{+}$is smaller than $\xi_{+}$for $\vartheta \lesssim 10^{\prime}$, and larger for larger

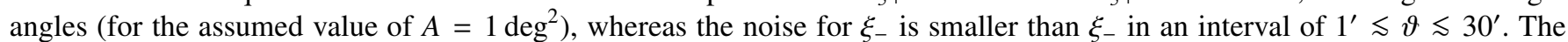
determination of $\xi_{\text {- }}$ on small angular scales is much more difficult than for $\xi_{+}$, owing to the smallness of $\xi_{\text {- }}$ for small $\vartheta$. Note that the noise scales like $A^{-1 / 2}$, so that from a survey of $16 \mathrm{sq} \mathrm{deg}$, like the DESCART survey (see van Waerbeke et al. 2001) one should be able to obtain reliable measurements of $\xi_{+}$for $\vartheta \lessgtr 1^{\circ}$, and of $\xi_{-}$for $10^{\prime \prime} \lessgtr \vartheta \lessgtr 2^{\circ}$ in bins of relative width of 0.1 .

\footnotetext{
${ }^{1}$ Whereas the Peacock \& Dodds (1996) prescription for the power spectrum yields fairly accurate results when compared with numerical simulations, a more accurate prescription has recently been given by Smith et al. (2002). We also note that approximate results for low-order cosmic shear statistics on small angular scales can be derived from the hierarchical ansatz, as in, e.g., Munshi \& Jain (2001). In addition, on very small angular scales one might wonder whether the weak lensing approximation - which replaces the reduced shear by the shear itself - remains valid; indications for its potential break-down may come from the results of the QSO-galaxy associations on small angular scales (e.g., Dolag \& Bartelmann 1997; Benitez et al. 2001; Menard \& Bartelmann 2002).
} 


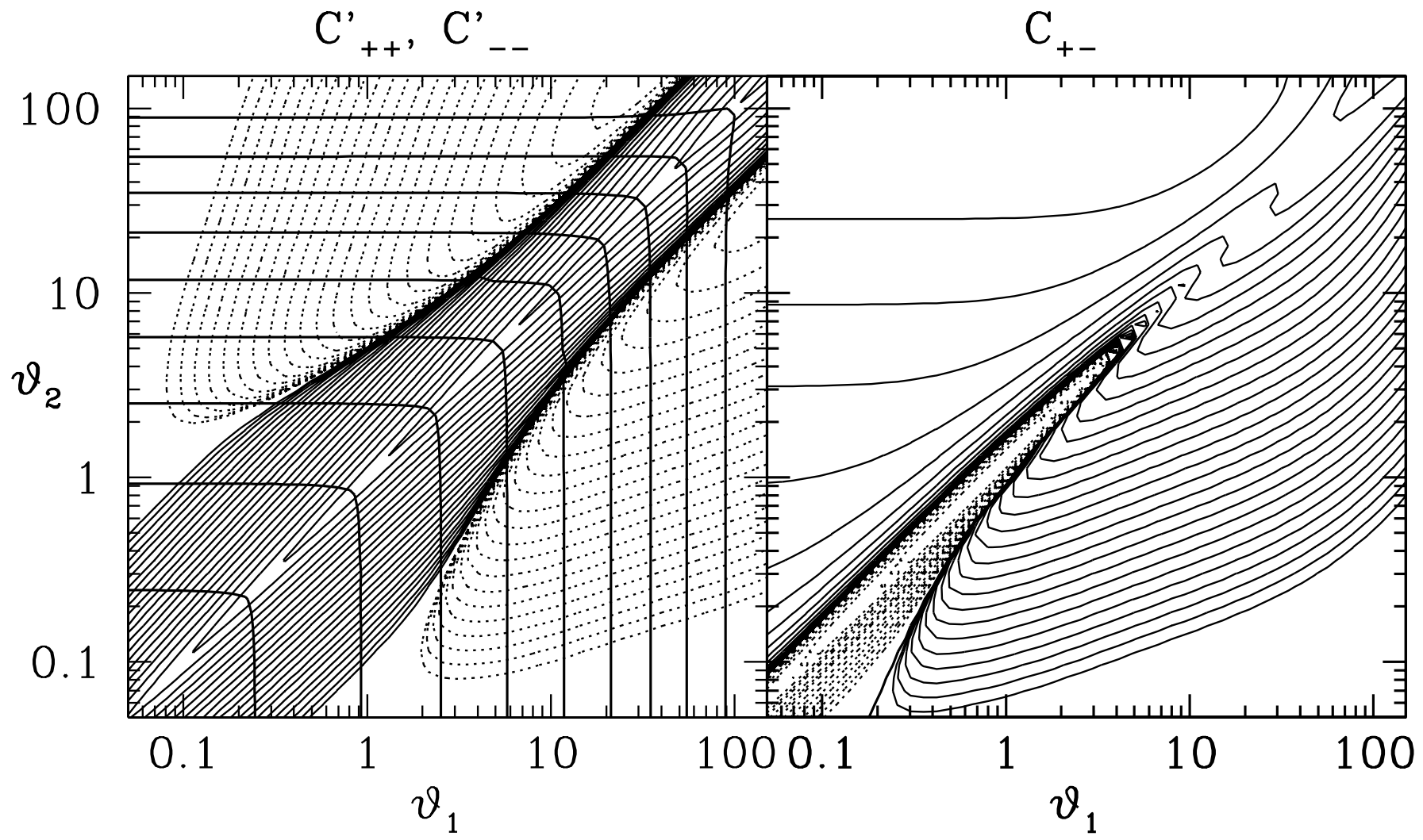

Fig. 1. The correlation functions. In the left panel, we have plotted the covariance matrices $\operatorname{Cov}_{++}^{\prime}\left(\vartheta_{1}, \vartheta_{2}\right)$ (thick solid curves) and $\operatorname{Cov}_{--}^{\prime}\left(\vartheta_{1}, \vartheta_{2}\right)$, i.e. the covariance matrices with the shot-noise term removed. For $\operatorname{Cov}_{++}^{\prime}\left(\vartheta_{1}, \vartheta_{2}\right)$, the contours are linearly spaced, with the lowest value at $10^{-9}$ (outer-most contour) and highest value $9 \times 10^{-9}$ for small $\vartheta_{1}, \vartheta_{2}$. For $\operatorname{Cov}_{--}^{\prime}\left(\vartheta_{1}, \vartheta_{2}\right)$, contours are logarithmically spaced, with consecutive contours differing by a factor 1.5 . The solid contours display positive values of $\operatorname{Cov}_{--}^{\prime}\left(\vartheta_{1}, \vartheta_{2}\right)$, starting from $10^{-14}$, with the maximum value of $\sim 3 \times 10^{-9}$ in the upper right corner, and dotted contours show negative values of $\operatorname{Cov}_{--}^{\prime}\left(\vartheta_{1}, \vartheta_{2}\right)$, starting at $-10^{-15}$. In the right panel, $\operatorname{Cov}_{+-}\left(\vartheta_{1}, \vartheta_{2}\right)$ is shown, again with logarithmically spaced contours differing by a factor of 1.5 . Solid contours are for positive values of $\operatorname{Cov}_{+-}\left(\vartheta_{1}, \vartheta_{2}\right)$, starting at $10^{-14}$, negative values are shown by dotted contours, starting at $-10^{-13}$.

Of course, the covariance of the shear will not only depend on the survey area, but also on its geometry; one might therefore design survey geometries which yield the desired noise behaviour as a function of angular scale (see, e.g., Kaiser 1998).

In order to show how strongly the correlation estimators at two angular scales are correlated, we define the correlation coefficient

$r_{\text {corr }}\left(\hat{\xi}_{ \pm} ; \vartheta_{1}, \vartheta_{2}\right):=\frac{\operatorname{Cov}^{\prime}\left(\hat{\xi}_{ \pm}, \vartheta_{1} ; \hat{\xi}_{ \pm}, \vartheta_{2}\right)}{\sqrt{\operatorname{Cov}^{\prime}\left(\hat{\xi}_{ \pm}, \vartheta_{1} ; \hat{\xi}_{ \pm}, \vartheta_{1}\right) \operatorname{Cov}^{\prime}\left(\hat{\xi}_{ \pm}, \vartheta_{2} ; \hat{\xi}_{ \pm}, \vartheta_{2}\right)}}$,

which is unity for $\vartheta_{1}=\vartheta_{2}$. Here, the prime indicates that the first term in the covariances [the one proportional to $\bar{\delta}\left(\vartheta_{1}-\vartheta_{2}\right)$ ] has been subtracted off, in order to show the correlation induced by cosmic shear. The correlation coefficient is plotted in the

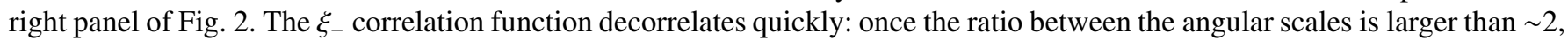
the correlation coefficient (39) has decreased to less than $10 \%$. In contrast to this, the correlation function $\xi_{+}$is correlated over much larger angular scales. This was expected, given that the filter function which relates the correlation function to the power spectrum is much broader for $\xi_{+}$than for $\xi_{-}$.

\section{Covariance of the aperture mass estimator}

Due to the particular interest in the aperture mass dispersion, we shall consider here the covariance matrix of the estimator (16),

$\operatorname{Cov}\left(\mathcal{M} ; \theta_{1}, \theta_{2}\right)=\left\langle\mathcal{M}\left(\theta_{1}\right) \mathcal{M}\left(\theta_{2}\right)\right\rangle-\left\langle M_{\mathrm{ap}}^{2}\left(\theta_{1}\right)\right\rangle\left\langle M_{\mathrm{ap}}^{2}\left(\theta_{2}\right)\right\rangle$. 

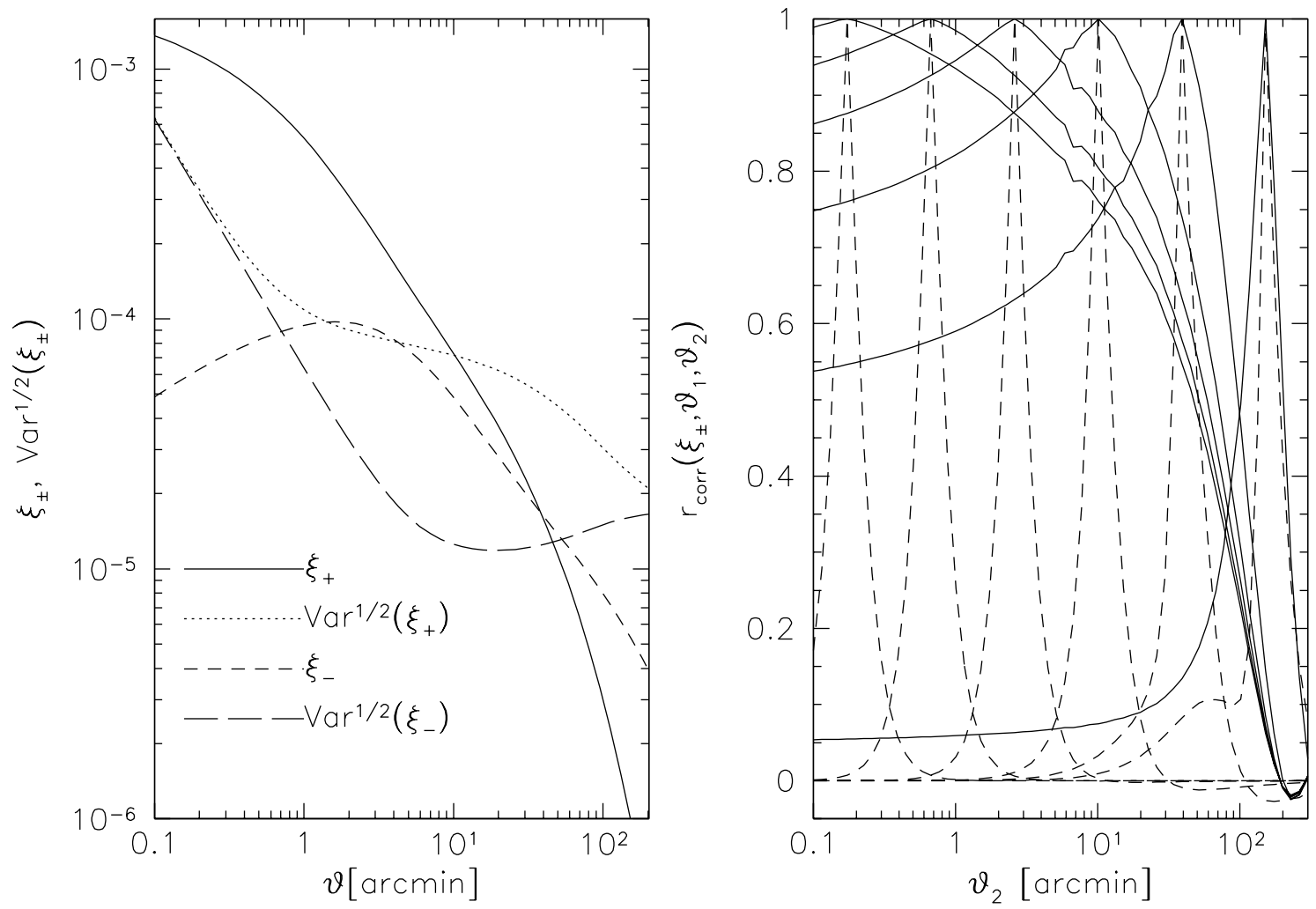

Fig. 2. Left panel: the square root of the variances $\sqrt{\operatorname{Var}\left(\hat{\xi}_{+} ; \vartheta\right)}$ and $\sqrt{\operatorname{Var}\left(\hat{\xi}_{-} ; \vartheta\right)}$ shown as dotted and long-dashed curves, together with the correlation functions $\xi_{+}(\vartheta)$ and $\xi_{-}(\vartheta)$ as solid and short-dashed curves, respectively. The model parameters are as described in the text; in particular, a fiducial value of the survey area of $1 \mathrm{deg}^{2}$ has been taken. For the diagonal part of the covariance matrix, we have assumed a relative bin size of $\Delta \vartheta / \vartheta=0.1$. For small $\vartheta$, the variance behaves as $\vartheta^{-1}$, as it is dominated by the noise from the intrinsic ellipticity of the source galaxies, i.e. the term $D(27)$, whereas for larger values of $\vartheta$, the main contribution comes from cosmic variance. Right panel: the correlation coefficient $r_{\text {corr }}$, as defined in (39), as a function of $\vartheta_{2}$, for various values of $\vartheta_{1}$. Solid curves show $r_{\text {corr }}\left(\hat{\xi}_{+}\right)$, dashed curves show $r_{\text {corr }}\left(\hat{\xi}_{-}\right)$. The value of $\vartheta_{1}$ corresponding to each curve can be read off from the point where a curve attains the value $r_{\text {corr }}=1$.

Munshi \& Coles (2000) have calculated the statistical properties of the aperture mass, using the hierarchical ansatz. We shall consider here the covariance in terms of the covariance of the two-point correlation function. Inserting (16) into this expression, the covariance matrix of $\mathcal{M}$ becomes

$$
\begin{aligned}
\operatorname{Cov}\left(\mathcal{M} ; \theta_{1}, \theta_{2}\right)= & \frac{(\Delta \vartheta)^{2}}{\theta_{1}^{2} \theta_{2}^{2}} \sum_{i=1}^{2 m_{1}} \sum_{j=1}^{2 m_{2}} \vartheta_{i} \vartheta_{j}\left\{K_{+}^{2} T_{+}\left(\frac{\vartheta_{i}}{\theta_{1}}\right) T_{+}\left(\frac{\vartheta_{j}}{\theta_{2}}\right) C_{++}\left(\vartheta_{i}, \vartheta_{j}\right)+\left(1-K_{+}\right)^{2} T_{-}\left(\frac{\vartheta_{i}}{\theta_{1}}\right) T_{-}\left(\frac{\vartheta_{j}}{\theta_{2}}\right) C_{--}\left(\vartheta_{i}, \vartheta_{j}\right)\right. \\
& \left.+K_{+}\left(1-K_{+}\right)\left[T_{+}\left(\frac{\vartheta_{i}}{\theta_{1}}\right) T_{-}\left(\frac{\vartheta_{j}}{\theta_{2}}\right) C_{+-}\left(\vartheta_{i}, \vartheta_{j}\right)+T_{-}\left(\frac{\vartheta_{i}}{\theta_{1}}\right) T_{+}\left(\frac{\vartheta_{j}}{\theta_{2}}\right) C_{+-}\left(\vartheta_{j}, \vartheta_{i}\right)\right]\right\}
\end{aligned}
$$

where we used the abbreviated notation $C_{++}\left(\vartheta_{1}, \vartheta_{2}\right) \equiv \operatorname{Cov}\left(\hat{\xi}_{+}, \vartheta_{1} ; \hat{\xi}_{+}, \vartheta_{2}\right)$ etc. The values of the aperture radius are taken to be $\theta_{k}=m_{k} \Delta \vartheta$ for $k=1,2$, and $\vartheta_{i}=(i-1 / 2) \Delta \vartheta$, as before. Hence, the covariance of the aperture mass dispersion can be obtained directly in terms of the covariances of the shear correlation functions. We can also obtain the ensemble-averaged covariance matrix; for this purpose, we separate the "delta-function" term in $C_{++}$and $C_{--}$from the rest and thus write $C_{++}\left(\vartheta_{1}, \vartheta_{2}\right)=$ $D \bar{\delta}\left(\vartheta_{1}-\vartheta_{2}\right)+C_{++}^{\prime}\left(\vartheta_{1}, \vartheta_{2}\right)$, similarily for $C_{--}$. Then the ensemble-average of (41) becomes

$$
\begin{aligned}
E\left(\operatorname{Cov}\left(\mathcal{M} ; \theta_{1}, \theta_{2}\right)\right)= & \frac{\sigma_{\epsilon}^{4}}{2 \pi A n^{2}} \int_{0}^{2 \min \left(\theta_{1}, \theta_{2}\right)} \frac{\mathrm{d} \vartheta \vartheta}{\theta_{1}^{2} \theta_{2}^{2}}\left[K_{+}^{2} T_{+}\left(\frac{\vartheta}{\theta_{1}}\right) T_{+}\left(\frac{\vartheta}{\theta_{2}}\right)+\left(1-K_{+}\right)^{2} T_{-}\left(\frac{\vartheta}{\theta_{1}}\right) T_{-}\left(\frac{\vartheta}{\theta_{2}}\right)\right] \\
& +\int_{0}^{2 \theta_{1}} \frac{\mathrm{d} \vartheta_{1} \vartheta_{1}}{\theta_{1}^{2}} \int_{0}^{2 \theta_{2}} \frac{\mathrm{d} \vartheta_{2} \vartheta_{2}}{\theta_{2}^{2}}\left\{K_{+}^{2} T_{+}\left(\frac{\vartheta_{1}}{\theta_{1}}\right) T_{+}\left(\frac{\vartheta_{2}}{\theta_{2}}\right) C_{++}^{\prime}\left(\vartheta_{1}, \vartheta_{2}\right)+\left(1-K_{+}\right)^{2} T_{-}\left(\frac{\vartheta_{1}}{\theta_{1}}\right) T_{-}\left(\frac{\vartheta_{2}}{\theta_{2}}\right) C_{--}^{\prime}\left(\vartheta_{1}, \vartheta_{2}\right)\right. \\
& \left.+K_{+}\left(1-K_{+}\right)\left[T_{+}\left(\frac{\vartheta_{1}}{\theta_{1}}\right) T_{-}\left(\frac{\vartheta_{2}}{\theta_{2}}\right) C_{+-}\left(\vartheta_{1}, \vartheta_{2}\right)+T_{-}\left(\frac{\vartheta_{1}}{\theta_{1}}\right) T_{+}\left(\frac{\vartheta_{2}}{\theta_{2}}\right) C_{+-}\left(\vartheta_{2}, \vartheta_{1}\right)\right]\right\}
\end{aligned}
$$

As expected, the covariance matrix of $\mathcal{M}$ does not depend on the binning of the correlation function. For the calculation of $\mathcal{M}$ for observational data, it is therefore best to choose very small bin widths, in order to minimize discretization errors. 
The first term in (42) yields the covariance in the absence of cosmic shear correlations, i.e. the covariance of the estimator $\mathcal{M}$ due to the intrinsic ellipticity of the source galaxies. This term can be written as

$$
\frac{\sigma_{\epsilon}^{4}}{2 \pi A n^{2} \theta_{2}^{2}} f\left(\theta_{1} / \theta_{2}\right)=3.939 \times 10^{-10}\left(\frac{\sigma_{\epsilon}}{0.3}\right)^{4}\left(\frac{A}{1 \mathrm{deg}^{2}}\right)^{-1}\left(\frac{n}{30 \operatorname{arcmin}^{-2}}\right)^{-2}\left(\frac{\theta_{2}}{1 \operatorname{arcmin}}\right)^{-2} f\left(\theta_{1} / \theta_{2}\right),
$$

where

$$
\begin{aligned}
f(R) & =\left(1-2 K_{+}+2 K_{+}^{2}\right) \int_{0}^{2} \mathrm{~d} x x T_{+}(x) T_{+}(R x)=\left(1-2 K_{+}+2 K_{+}^{2}\right) \int_{0}^{2} \mathrm{~d} x x T_{-}(x) T_{-}(R x) \\
& =\left(1-2 K_{+}+2 K_{+}^{2}\right)(576)^{2} \int_{0}^{\infty} \frac{\mathrm{d} t}{R^{4} t^{7}} J_{4}^{2}(R t) J_{4}^{2}(t)
\end{aligned}
$$

where the explicit relation has been obtained by making use of the original definitions of $T_{ \pm}$. The function $f(R)$ is maximized at $R=1$, where its value is $f(1) \approx 0.29$, and it decreases quickly for appreciable ratios of the aperture radii. The dependence on $K_{+}$ is simple, and obviously this noise term is minimized for $K_{+}=1 / 2$, i.e. when both correlation functions enter the estimate $\mathcal{M}$ with equal weight. The variance of the estimator $\mathcal{M}$ in the absence of shear correlations can be compared with the corresponding expression obtained in SvWJK, obtained from their Eqs. (5.12) and (5.16), yielding

$$
\frac{G^{2} \sigma_{\epsilon}^{4}}{2 \pi^{2} n^{2} \theta^{4} N_{\mathrm{f}}}=\frac{2.88}{\pi^{2}} \frac{\sigma_{\epsilon}^{4}}{A n^{2} \theta^{2}}
$$

where $G=1.2$ is a numerical coefficient (calculated from the filter function in the definition of $M_{\text {ap }}$ ), and $N_{\mathrm{f}}$ is the number of independent apertures that can be placed on the data field, taken to be $A /\left(4 \theta^{2}\right)$. Together with $f(1) \approx 0.29$, one clearly sees that the variance of the estimator $\mathcal{M}$ is substantially smaller than that used in SvWJK which was based on placing non-overlapping apertures on the data field. The main reason for this difference is that in the latter method, only one component of the source ellipticities is used; furthermore, apertures separated by less than their diameter are statistically nearly independent. Hence, the determination of $\left\langle M_{\mathrm{ap}}^{2}\right\rangle$ through the shear correlation function is not only more practical in the presence of gaps in the data field, but also far more efficient than the alternative method.

We have plotted in Fig. 3 the square root of the variance $\operatorname{Var}(\mathcal{M}, \theta) \equiv \operatorname{Cov}(\mathcal{M} ; \theta, \theta)$ of $\mathcal{M}$, both for the case of no correlations, and for our standard model for the cosmic shear. For small angular scales, the variance is completely dominated by the intrinsic source ellipticities, whereas the cosmic variance is the important noise error for larger angular scales. For the parameters used here, the transition between these two regimes occurs at a few arcminutes. It must be noted that the shape of the variance curves are independent of the survey area $A$. The results shown in Fig. 3 can be seen as an a posteriori justification of using the Gaussian assumption for the calculation of the shear four-point function in Sect. 4. In Fig. 4 of van Waerbeke et al. (2002), the influence of the non-linear density evolution on the kurtosis of $M_{\text {ap }}$ was studied, using ray-tracing simulations through numerically generated cosmological matter distributions. The non-Gaussian effects start to become non-negligible for angular scales below $\sim 5^{\prime}$. As can be seen in Fig. 3, this is about the scale where the transition occurs between the variance being dominated by the intrinsic ellipticity distribution and the cosmic variance. Hence, we can expect that a more advanced treatment of the shear four-point function would yield a slightly larger variance in this transition region around $\sim 5^{\prime}$ : for significantly smaller scales, it is dominated by the intrinsic ellipticity noise, and for larger scales, the shear four-point function is basically Gaussian.

The variances of $\mathcal{M}$ for the cases $K_{+}=0$ and $K_{+}=1$ are basically identical, and larger by a factor $\sim 2$ than the variance of the estimator for $K_{+}=1 / 2$. Hence, to minimize the variance of the estimator $\mathcal{M}, K_{+}=1 / 2$ should be chosen. With this choice, the results are unchanged even in the presence of a $B$-mode contribution (see SvWM). As was already mentioned by $C 02$, using

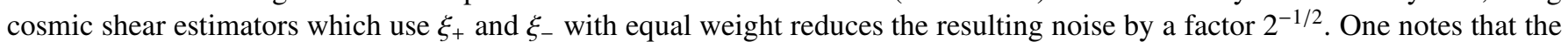
variance for large $\theta$ rises, but very slowly. We can compare the behavior of the variance of $\mathcal{M}$ with that derived in $\mathrm{SvWJK}$ for a more direct estimator for the aperture mass dispersion; using Eqs. (5.12) and (5.16) of that paper, one finds in the limit of large angles (and, to make the estimate comparable to the one obtained here, zero kurtosis) that $\sqrt{\operatorname{Var}\left(\mathcal{M}^{\prime} ; \theta\right)} \approx 2 \theta\left\langle M_{\text {ap }}^{2}\right\rangle(\theta) / A$, where $\mathcal{M}^{\prime}$ is the estimator used in SvWJK. The functional behavior with $\theta$ is similar to that seen in Fig. 3, but the amplitude is lower by a factor of about 2 for $K_{+}=0,1$, and about 3 for $K_{+}=1 / 2$; this again shows the superiority of the estimator considered here in comparison to laying down independent apertures on the data field.

To investigate the correlation of the estimator $\mathcal{M}$ between different angular scales, we define the correlation coefficient

$r_{\text {corr }}\left(\mathcal{M} ; \theta_{1}, \theta_{2}\right):=\frac{\operatorname{Cov}\left(\mathcal{M} ; \theta_{1}, \theta_{2}\right)}{\sqrt{\operatorname{Cov}\left(\mathcal{M} ; \theta_{1}, \theta_{1}\right) \operatorname{Cov}\left(\mathcal{M} ; \theta_{2}, \theta_{2}\right)}}$,

which has the property that $r_{\text {corr }}(\mathcal{M} ; \theta, \theta)=1$. The dependence of this correlation on the ratio of the angular scales then provides information on the correlated error of the determination of the aperture mass dispersion on different angular scales. In the right panel of Fig. 3 we have plotted this correlation coefficient as a function of $\theta_{2}$, for various values of $\theta_{1}$. The logarithmic representation clearly shows that this correlation coefficient depends mainly on the ratio $\theta_{1} / \theta_{2}$. The correlation drops off quickly, 

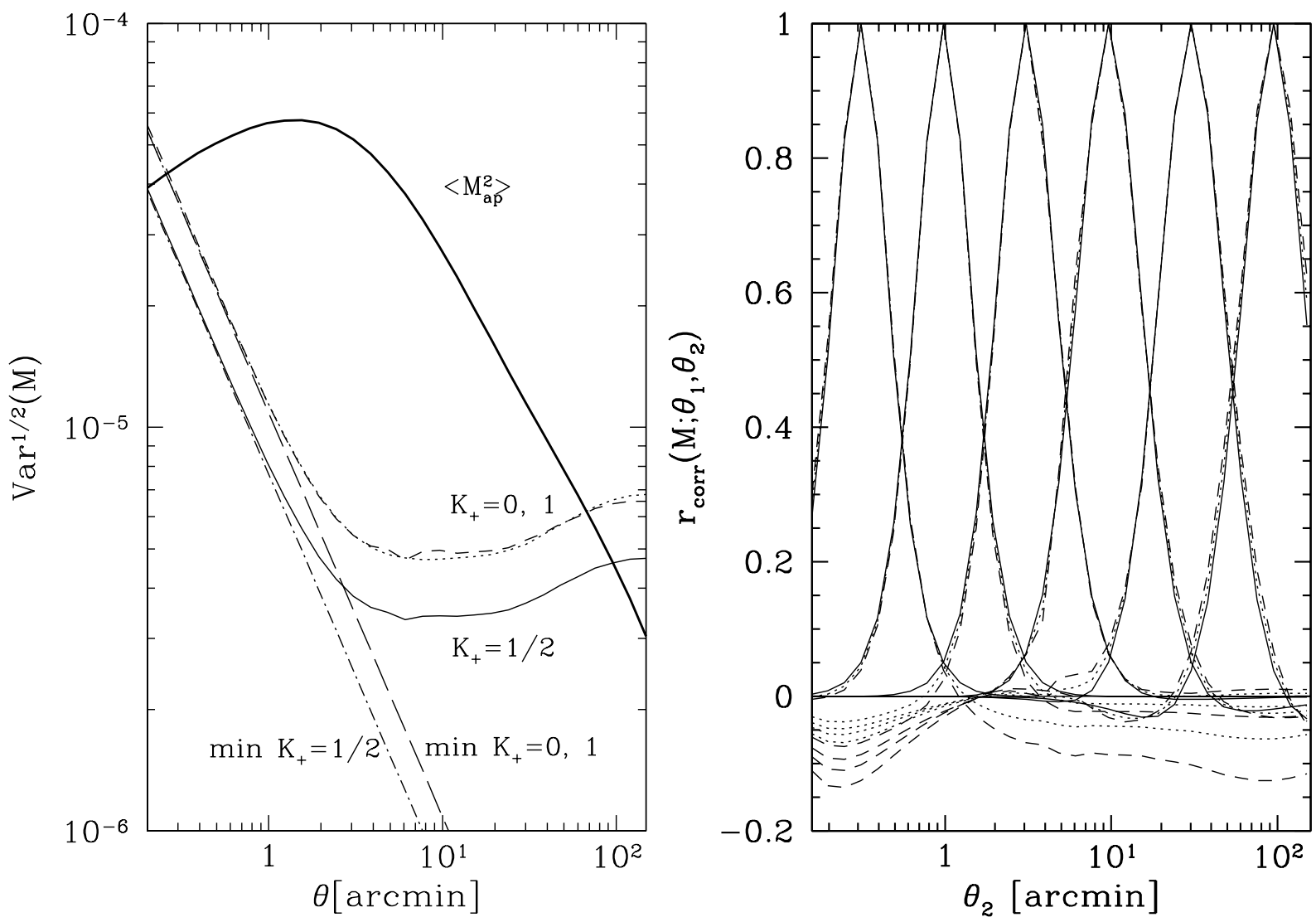

Fig. 3. Left panel: the square-root of the autovariance of $\mathcal{M}$ as a function of angular scale. The long-dashed and dash-dotted curves show the minimum variance, i.e. in the absence of a shear correlation; this variance is due solely to the intrinsic ellipticity of source galaxies. Curves are shown for $K_{+}=0,1 / 2,1$, where the minimum variance is the same for $K_{+}=0$ and 1 . The solid, dashed and dotted curves show the variance in the presence of a cosmic shear; also here, the cases $K_{+}=0$ and $K_{-}=1$ are nearly the same, and the variance is smallest for $K_{+}=1 / 2$. For comparison, $\left\langle M_{\mathrm{ap}}^{2}\right\rangle(\theta)$ is plotted as thick solid curve. As for the other figures shown before, our standard set of parameters $A=1 \mathrm{deg}^{2}$, $n=30 \operatorname{arcmin}^{-2}$ and $\sigma_{\epsilon}=0.3$ has been used; the variance scales as $A^{-1}$. Right panel: the correlation coefficient $r_{\text {corr }}\left(\mathcal{M} ; \theta_{1}, \theta_{2}\right)$ of the covariance of the estimator $\mathcal{M}$ is plotted as a function of $\theta_{2}$, for various values of $\theta_{1}$; the values of $\theta_{1}$ can be localized as those points where the correlation function attains the value unity. The solid curves are for $K_{+}=0$, i.e. when only the correlation function $\xi_{-}$is used in the estimate of $\mathcal{M}$, the dotted curves are for $K_{+}=1 / 2$, and the dashed curves for $K_{+}=1$. The width of all three families of curves is very similar and (in logarithmic terms) basically independent of $\theta_{1}$. The $K_{+}=0$ curves do not develop a tail of anticorrelation, as is the case for $K_{+}=1$ (and therefore also for $K_{+}=1 / 2$ ). Hence, whereas $K_{+}=1 / 2$ yields the smallest variance of the estimator $\mathcal{M}$, it leads to a small but long-range correlation between different angular scales.

so that estimates of $\left\langle M_{\mathrm{ap}}^{2}\right\rangle(\theta)$ for two angles with ratio $\theta_{1} / \theta_{2} \lesssim 1 / 3$ or $\theta_{1} / \theta_{2} \gtrsim 3$ are practically uncorrelated. This was to be expected given that $\left\langle M_{\mathrm{ap}}^{2}\right\rangle(\theta)$ is obtained from the power spectrum $P_{K}(\ell)$ through a very well localized filter function. Hence, the estimator $\mathcal{M}$ decorrelates much faster than those of the shear correlation functions. Also seen in Fig. 3 is the fact that in the case of $K_{+}=1 / 2$ and $K_{+}=1$, the correlation coefficient attains long negative, but low-amplitude tails, whereas they are basically absent if $K_{+}=0$. This is due to the much faster decorrelation of $\xi_{-}$with scale ratio than that of $\xi_{+}$.

\section{A simple estimator for the power spectrum, and its covariance}

The relations (5) may be used to define an estimator for the power spectrum $P_{K}(\ell)$ as

$\hat{P}(\ell)=2 \pi \int_{\theta_{\min }}^{\theta_{\max }} \mathrm{d} \theta \theta\left[K_{+} \xi_{+}(\theta) J_{0}(\ell \theta)+\left(1-K_{+}\right) \xi_{-}(\theta) J_{4}(\ell \theta)\right]$

where $K_{+} \in[0,1]$ again describes the relative contribution from the $\xi_{+}$correlation. Here, $\theta_{\min }$ and $\theta_{\max }$ describe the range over which the correlation function has been measured. If this range extends from zero to infinity, the estimator (45) would be unbiased (and would yield the $E$-mode power spectrum for $K_{+}=1 / 2$ even in the presence of $B$-modes); for real datasets, where this range is finite, (45) is a biased estimator. Note that in the absence of $B$-modes, Eq. (45) remains valid even if the factor $K_{+}$is chosen to 


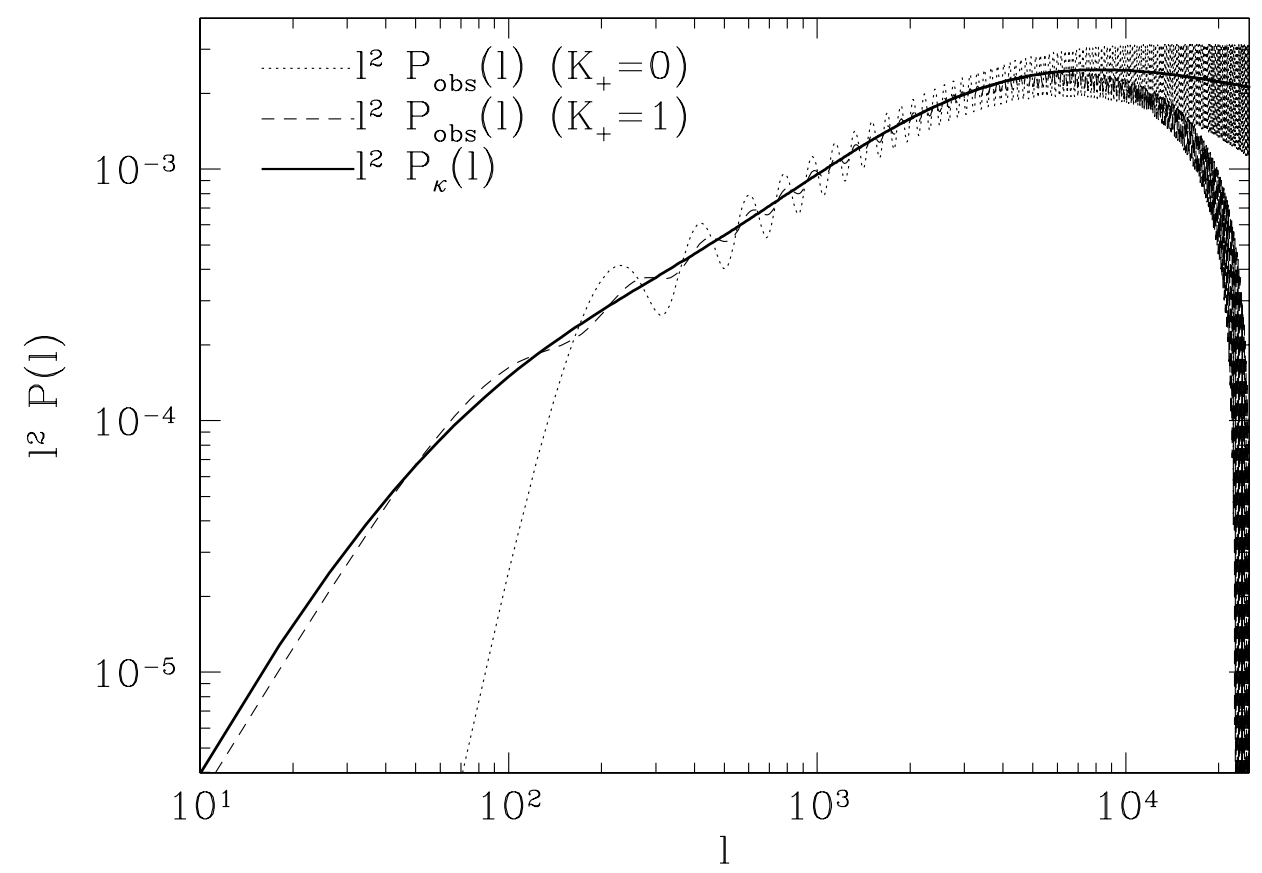

Fig. 4. The thick solid line displays the dimensionless projected power spectrum $\ell^{2} \mathcal{P}_{\kappa}(\ell)$, whereas the other two curves show the "observed"

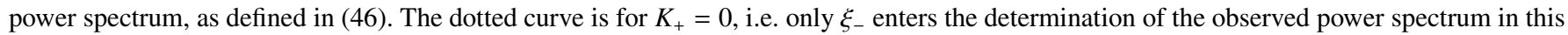
case; the dashed curve is for $K_{+}=1$. In this plot is was assumed that the correlation functions are known between $\theta_{\min }=6^{\prime \prime}$ and $\theta_{\max }=2^{\circ}$.

be a function of $\ell$. The expectation value can be obtained by inserting the relation (2) between the correlation functions and the true power spectrum into (45) to yield

$\langle\hat{P}(\ell)\rangle \equiv P_{\mathrm{obs}}(\ell)=\int_{0}^{\infty} \mathrm{d} \ell^{\prime} \ell^{\prime}\left[K_{+} G_{0}\left(\ell, \ell^{\prime}\right)+\left(1-K_{+}\right) G_{4}\left(\ell, \ell^{\prime}\right)\right] P_{K}\left(\ell^{\prime}\right)$,

with

$G_{n}\left(\ell, \ell^{\prime}\right)=\int_{\theta_{\min }}^{\theta_{\max }} \mathrm{d} \theta \theta J_{n}(\ell \theta) J_{n}\left(\ell^{\prime} \theta\right)=\left[\frac{\theta}{\ell^{\prime 2}-\ell^{2}}\left\{\ell^{\prime} J_{n+1}\left(\ell^{\prime} \theta\right) J_{n}(\ell \theta)-\ell J_{n}\left(\ell^{\prime} \theta\right) J_{n+1}(\ell \theta)\right\}\right]_{\theta=\theta_{\min }}^{\theta=\theta_{\max }}$.

We have plotted the "observed" power spectrum as $\ell^{2} P_{\text {obs }}(\ell)$ in Fig. 4 , for $K_{+}=0$ and $K_{+}=1$, assuming that $\theta_{\text {min }}=6$ " and $\theta_{\max }=2^{\circ}$. A comparison with the underlying power spectrum (shown as heavy solid curve) shows that $P_{\text {obs }}$ traces the true power spectrum over a wide range of $\ell$-values, though in an oscillatory way. If $P_{\text {obs }}$ is determined solely from $\xi_{-}$(i.e. $K_{+}=0$ ), it substantially underestimates the power for $\ell \lesssim 10^{2}$ (that is, approximately for $\ell \lesssim 2 \pi / \theta_{\max }$ ), but traces the true power spectrum out to the largest values of $\ell$ plotted. Conversely, the observed power determined from $\xi_{+}$yields good estimates of the true power for small values of $\ell$, but deviates from it strongly for $\ell \geq 8 \times 10^{3}$, that is for values of $\ell$ much less than $2 \pi / \theta_{\text {min }} \sim 2 \times 10^{5}$. The different behavior of the two estimates again is due to the different filter function through which correlation function and power spectrum are related. Figure 4 suggests that the best estimate for the power spectrum is obtained by choosing $K_{+}=1$ for small values of $\ell$, and $K_{+}=0$ for large $\ell$.

The covariance matrix of $\hat{P}$ reads, for $K_{+}=1$,

$\operatorname{Cov}\left(\hat{P} ; \ell, \ell^{\prime}\right)=(2 \pi)^{2} \int_{\theta_{\min }}^{\theta_{\max }} \mathrm{d} \theta \theta J_{0}(\ell \theta) \int_{\theta_{\min }}^{\theta_{\max }} \mathrm{d} \theta^{\prime} \theta^{\prime} J_{0}\left(\ell^{\prime} \theta^{\prime}\right) C_{++}\left(\theta, \theta^{\prime}\right) ;$

the generalization for other values of $K_{+}$is obvious and shall not be reproduced here.

To estimate the power spectrum from cosmic shear data, it is useful to define the power in a band with upper and lower $\ell$-values $\ell_{i \mathrm{u}}$ and $\ell_{i \mathrm{l}}$ as

$\mathcal{P}_{i}:=\frac{1}{\Delta_{i}} \int_{\ell_{i 1}}^{\ell_{i \mathrm{u}}} \mathrm{d} \ell \ell \hat{P}(\ell)=\frac{2 \pi}{\Delta_{i}} \int_{\theta_{\min }}^{\theta_{\max }} \frac{\mathrm{d} \theta}{\theta}\left\{K_{+} \xi_{+}(\theta)\left[g_{+}\left(\ell_{i \mathrm{u}} \theta\right)-g_{+}\left(\ell_{i l} \theta\right)\right]+\left(1-K_{+}\right) \xi_{-}(\theta)\left[g_{-}\left(\ell_{i \mathrm{u}} \theta\right)-g_{-}\left(\ell_{i l} \theta\right)\right]\right\}$,

where $\Delta_{i}=\ln \left(\ell_{i \mathrm{u}} / \ell_{i 1}\right)$ is the logarithmic width of the band, and

$g_{+}(x)=x J_{1}(x) ; \quad g_{-}(x)=\left(x-\frac{8}{x}\right) J_{1}(x)-8 J_{2}(x)$. 


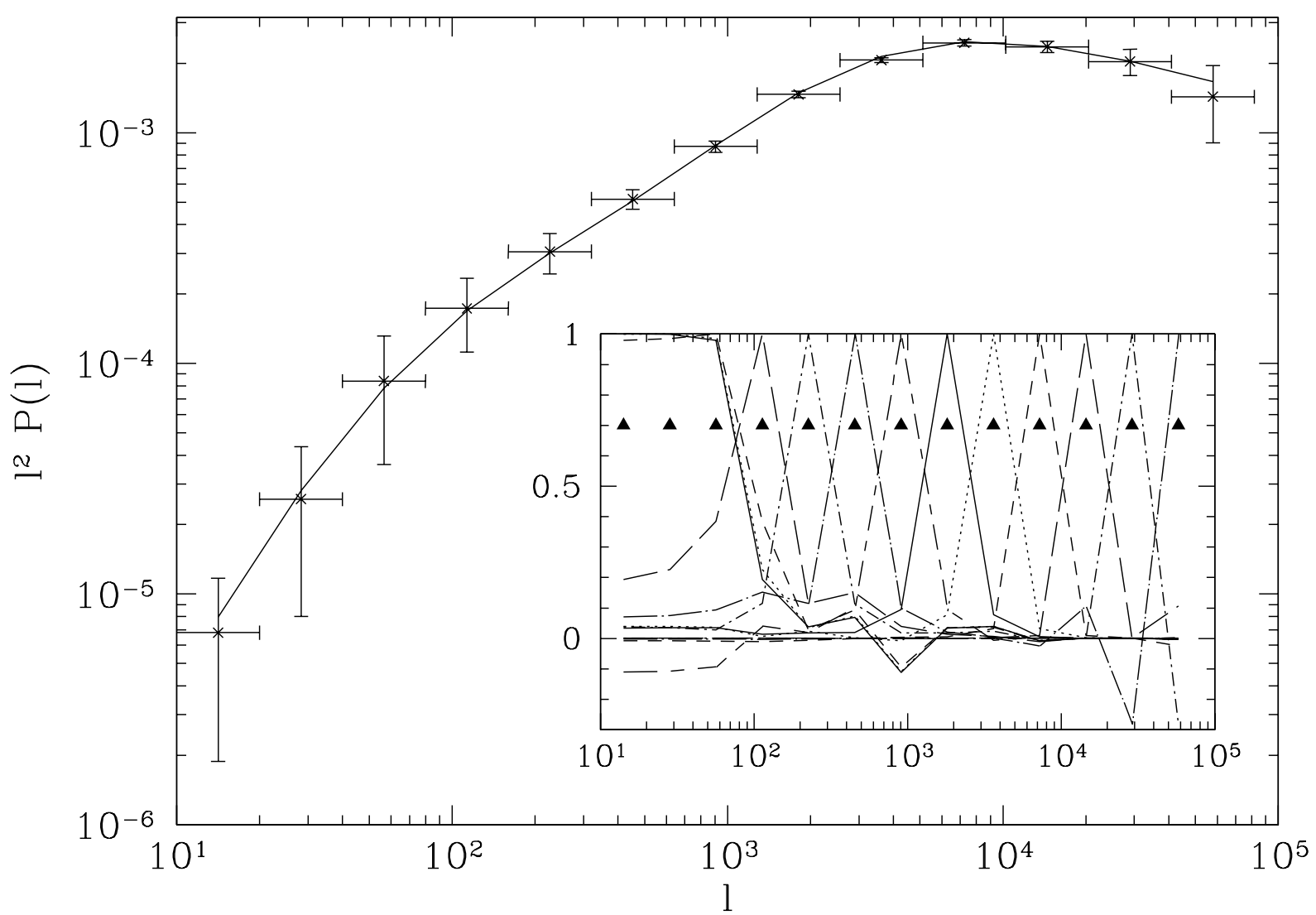

Fig. 5. The large panel shows the estimates of the band power, shown as horizontal bars whose length indicates the bins used. The error bar on each bin shows the square root of the autovariance of the band power, and the solid curve is the underlying power spectrum, $\ell^{2} P_{k}(\ell)$. For this figure, we have assumed that the correlation functions are measured for $6^{\prime \prime} \leq \vartheta \leq 2^{\circ}$, from a survey of $A=25 \mathrm{deg}^{2}$. The inset figure shows the correlation coefficient between the 13 different bins, where the triangles indicate the center $\bar{\ell}$ of each bin. One sees that the bands are very little correlated, except for the three bins with smallest $\ell$; in fact, the first three band power estimates are fully correlated. This explains why the band-power estimator yields reasonable results even for $\ell<2 \pi / \theta_{\max } \sim 180-$ this is just a coincidence.

One expects that the band power traces $\bar{\ell}_{i}^{2} P_{K}\left(\bar{\ell}_{i}\right)$, where $\bar{\ell}_{i}$ is the geometric mean of $\ell_{i \mathrm{u}}$ and $\ell_{i l}$, i.e. the center of the bin. The covariance of the band power of two bins $i$ and $j$ is

$$
\begin{aligned}
\operatorname{Cov}\left(\mathcal{P}_{i} \mathcal{P}_{j}\right)= & \frac{2 \pi \sigma_{\epsilon}^{4}}{\Delta_{i} \Delta_{j} A n^{2}} \int_{\theta_{\min }}^{\theta_{\max }} \frac{\mathrm{d} \theta}{\theta^{3}}\left[K_{+}^{2} G_{i+}(\theta) G_{j+}(\theta)+\left(1-K_{+}\right)^{2} G_{i-}(\theta) G_{j-}(\theta)\right] \\
& +\frac{(2 \pi)^{2}}{\Delta_{i} \Delta_{j}} \int_{\theta_{\min }}^{\theta_{\max }} \frac{\mathrm{d} \theta}{\theta} \int_{\theta_{\min }}^{\theta_{\max }} \frac{\mathrm{d} \theta^{\prime}}{\theta^{\prime}}\left\{K_{+}^{2} C_{++}^{\prime}\left(\theta, \theta^{\prime}\right) G_{i+}(\theta) G_{j+}\left(\theta^{\prime}\right)+\left(1-K_{+}\right)^{2} C_{--}^{\prime}\left(\theta, \theta^{\prime}\right) G_{i-}(\theta) G_{j-}\left(\theta^{\prime}\right)\right. \\
& \left.+K_{+}\left(1-K_{+}\right) C_{+-}\left(\theta, \theta^{\prime}\right)\left[G_{i+}(\theta) G_{j-}\left(\theta^{\prime}\right)+G_{i-}\left(\theta^{\prime}\right) G_{j+}(\theta)\right]\right\}
\end{aligned}
$$

where $G_{i \pm}(\theta)=g_{ \pm}\left(\ell_{i \mathrm{u}} \theta\right)-g_{ \pm}\left(\ell_{i 1} \theta\right)$. In (51), we have already split off the "delta-function" part of the correlation covariance matrices, which yields the first term. It should be noted that the foregoing expressions remain valid if $K_{+}$is varied as a function of $\ell$. From Fig. 3 it is clear that in order to get the least bias, one wants to choose $K_{+} \sim 1$ for small $\ell$, and $K_{+} \sim 0$ for large $\ell$; for the intermediate region, setting $K_{+}=1 / 2$ should yield the smallest error on the power spectrum. We have therefore constructed a function $K_{+}(\ell)$ which has these desired properties.

In Fig. 5 we have plotted the band power for our reference model parameters, in 13 bins of width $\ell_{i \mathrm{u}} / \ell_{i 1}=2$, between $\ell=10$ and $\ell \approx 8 \times 10^{4}$. The band power is shown as crosses, and vertical error bars show the range of the bins. For comparison, the solid curve shows $\ell^{2} P_{k}(\ell)$; as expected, with this new choice of $K_{+}(\ell)$, the band power traces the underlying power spectrum over a very wide range of wavenumbers. Only in the bins with the smallest and largest value of $\ell$ is there a significant bias; over the range $2 \pi / \theta_{\max } \approx 180 \lesssim \ell \lesssim 2 \pi / \theta_{\min } \approx 2 \times 10^{5}$, the band power estimator is practically unbiased. Next we calculated the error bars on the band power, by taking the square root of the diagonal part of (51). For this calculation, we have assumed to have a total area of $A=25 \mathrm{deg}^{2}$, for which the condition $\theta_{\max }^{2} \ll A$ for the validity of the treatment of the ensemble average in Sect. 5 is approximately satisfied. The square root of this autovariance is plotted as errorbars on the band power in Fig. 5; as can be seen from this figure, the signal-to-noise ratio is larger than unity in all bins shown, and in fact very large for intermediate values of $\ell$. Hence, the power spectrum $P_{\kappa}(\ell)$ can be measured over a broad range of $\ell$ for the parameters chosen here. 
Of course, in order to interpret the error bars correctly, it is important to see the degree of correlated noise between different bands. The correlation matrix for the bins (defined in full analogy to (44)) was calculated and its values are plotted in the inset of Fig. 5. One sees that errors of the bins for intermediate and high values of $\ell$ are essentially uncorrelated (the correlation coefficient for neighboring bins is $\lesssim 10 \%$ for $\ell \gtrsim 200$ ); however, for $\ell \lesssim 100$ the bins become strongly correlated. In fact, the agreement of the band powers with the underlying power spectrum is forticious for $\ell \lesssim 100$ : the three band powers at lowest $\ell$ are nearly fully correlated, so that these three points contain practically the same information of the underlying power spectrum.

The method presented here for the determination of the power spectrum has the virtue of its simplicity. Other methods for determining the power spectrum from shear data have been investigated, e.g. by Kaiser (1998), Seljak (1998) and Hu \& White (2001). Our approach has the property that it makes use only of the shear correlation functions, not on the spatial distribution of the shear. Since the shear correlation function contains all two-point statistical information of the shear field, no information loss occurs. Comparing the results of Fig. 5 with those of Hu \& White (2001) it seems that both methods yield very similar error bars of the power spectrum, and that in the respective $\ell$-range of applicability, the decorrelation between neighboring bins is equally quick. Since our method does not require the "pixelization" of shear data, it can estimate the power spectrum to larger values of $\ell$.

\section{Constraints on cosmological parameters}

One of the central goals of cosmic shear research is the determination of cosmological parameters. Since the power spectrum $P_{\kappa}$, and thus the shear correlation functions, depend on the cosmological model, precise measurements of cosmic shear can be used to tie down the range of allowable model parameters (e.g., Jain \& Seljak 1997; Bernardeau et al. 1997). The largest cosmic shear survey today already yielded significant model constraints (van Waerbeke et al. 2001, 2002; Hoekstra et al. 2002). We shall briefly discuss the expected confidence regions in parameter space, using the previously calculated covariance matrix of the shear correlation functions. For that, we consider a figure-of-merit function

$\chi^{2}(p):=\sum_{i j}\left(\xi_{i}(p)-\xi_{i}^{\mathrm{t}}\right) \operatorname{Cov}_{i j}^{-1}\left(\xi_{j}(p)-\xi_{j}^{\mathrm{t}}\right)$,

where the index ${ }^{\mathrm{t}}$ indicates the correlation function of the input model, $p$ is a set of model parameters, and the summation indices label the angular bins of the correlation function. We shall consider three different kinds of the function (52): in the first, named $\chi_{+}^{2}$ hereafter, the correlation functions in (52) are the $\xi_{+}$correlations, and the covariance matrix corresponds to (23). The second kind is denoted by $\chi_{-}^{2}$ and uses the $\xi_{-}$correlation function. The third kind, $\chi_{\text {tot }}^{2}$, is obtained by constructing a vector $\xi_{i}=\left(\xi_{+1}, \xi_{+2}, \ldots, \xi_{+N}, \xi_{-1}, \xi_{-2}, \ldots, \xi_{-N}\right)$, when there are $N$ angular bins in which $\xi_{ \pm}$has been measured. Correspondingly, the covariance matrix in (52) is the $2 \times 2$-block matrix with $C_{++}$and $C_{--}$in the upper left and lower right quadrant, respectively, and $C_{+-}$in the other two.

\subsection{The $\sigma_{8}-\Omega_{m}$ degeneracy}

We first have calculated $\chi^{2}$ for a two-parameter set of cosmological models, by varying $\Omega_{\mathrm{m}}$ and $\sigma_{8}$; these two parameters were considered before in van Waerbeke et al. (2001). We kept the other cosmological parameters fixed, except that flat Universes are considered, i.e. $\Omega_{\mathrm{m}}+\Omega_{\Lambda}=1$. From the confidence contours plotted in van Waerbeke et al., it is anticipated that the $\chi^{2}$-function, considered as a function of $\Omega_{\mathrm{m}}$ and $\sigma_{8}$, will have a long and curved valley close to its minimum. This was verified here. It is therefore useful to consider the combination $\Sigma_{8}:=\sigma_{8}\left[0.41+0.59\left(\Omega_{\mathrm{m}} / 0.3\right)^{-0.68}\right]^{-1}$ as parameter when plotting contours of $\chi^{2}$ (the numerical values occurring in this definition have been obtained by a fit through the valley line in the $\Omega_{\mathrm{m}}-\sigma_{8}$ plane).

In Fig. 6 we have plotted contours of constant $\chi^{2}$ in the $\Omega_{\mathrm{m}}-\Sigma_{8}$ plane, corresponding to 1- $\sigma, 2-\sigma$ and 3- $\sigma$ confidence regions. In the left panel, we kept the shape parameter of the power spectrum fixed, $\Gamma=0.21$, whereas in the right panel we used $\Gamma=$ $0.7 \Omega_{\mathrm{m}}$, adequate for a (dimensionless) Hubble constant of $h=0.7$. Shown are the confidence regions for all three functions $\chi^{2}$, as indicated. The first point to note is that, for a given value of $\Omega_{\mathrm{m}}, \sigma_{8}$ is very well constrained, to within a few percent. This implies that the normalization of the power spectrum is very well determined from cosmic shear observations. Secondly, the 1- $\sigma$ uncertainty on $\Omega_{\mathrm{m}}$ is about 0.1 for an assumed survey size of $A=5 \mathrm{deg}^{2}$; indeed, the left panel of Fig. 6 can be compared directly with similar figures in van Waerbeke et al., and the constraints on $\Omega_{\mathrm{m}}$ are quite similar. Third, if the shape parameter changes with $\Omega_{\mathrm{m}}$, the confidence regions are narrower than when setting $\Gamma=0.21$ as constant, which implies that the shear correlation functions are sensitive measures for $\Gamma$.

\subsection{Additional parameters}

In the prospect of measuring the cosmological parameters, we must also allow for our lack of knowledge of some parameters which might have a significant effect on our observable, but which are poorly constrained. In particular, it is important to explore the possible directions of degeneracies and to identify which combination of parameters offers the most promising measurements. 


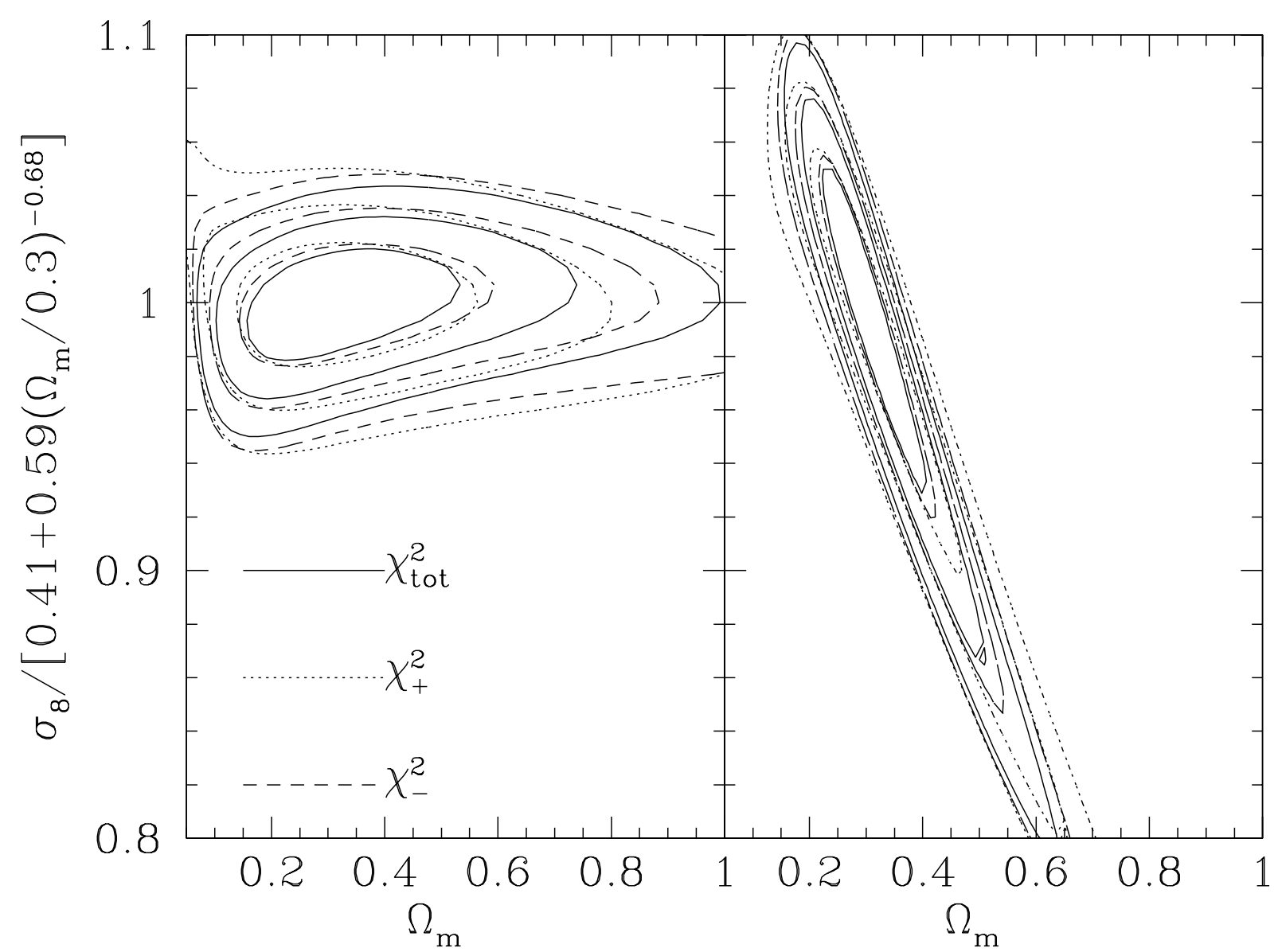

Fig. 6. 1- $\sigma, 2-\sigma$ and 3- $\sigma$ confidence contours in the $\Omega_{\mathrm{m}}-\Sigma_{8}$-plane, where $\Sigma_{8}$ is a scaled version of the power spectrum normalization parameter $\sigma_{8}$, as indicated. Dotted, dashed and solid contours correspond to $\chi_{+}^{2}, \chi_{-}^{2}$ and $\chi_{\text {tot }}^{2}$. In the left panel, the shape parameter of the power spectrum $\Gamma=0.21$, whereas in the right panel, $\Gamma=0.7 \Omega_{\mathrm{m}}$. The reference model is the one used before, i.e. $\Omega_{\mathrm{m}}=0.3, \Omega_{\Lambda}=1-\Omega_{\mathrm{m}}, \sigma_{8}=1$. The contours are obtained by assuming a survey area of $A=5 \mathrm{deg}^{2}$, and that the correlation functions were measured in the range $12^{\prime \prime} \leq \vartheta \leq 30^{\prime}$.

As shown in Jain \& Seljak (1997), the shear second-order statistics depend primarily on four parameters: $\Omega_{\mathrm{m}}, \sigma_{8}, \Gamma$ and $z_{\mathrm{s}}$, the source redshift parameter. We now model the source redshift distribution with

$p(z)=\frac{\beta}{z_{\mathrm{s}} \Gamma\left(\frac{1+\alpha}{\beta}\right)}\left(\frac{z}{z_{\mathrm{s}}}\right)^{\alpha} \exp \left[-\left(\frac{z}{z_{\mathrm{s}}}\right)^{\beta}\right]$,

where we choose $\alpha=2$ and $\beta=1.2$, and take $z_{\mathrm{s}}$ as the free parameter; note that the mean redshift of the sources is $\langle z\rangle \approx$ $2.09 z_{\mathrm{s}}$. This choice is motivated from observational considerations (see van Waerbeke et al. 2002). We therefore constructed the 4-dimensional figure-of-merit function (52), where now $\xi_{i} \equiv \xi_{+}\left(\theta_{i} ; \Omega_{\mathrm{m}}, \sigma_{8}, \Gamma, z_{\mathrm{s}}\right)$. The fiducial model is chosen to be $\Omega_{\mathrm{m}}=0.3$, $\sigma_{8}=1, \Gamma=0.21$ and $z_{\mathrm{s}}=0.44$. It is assumed that the shear correlation function is measured in the range $0^{\prime} 6 \leq \vartheta \leq 30^{\prime}$, comparable with the most recent cosmic shear measurements. We also fix the survey area to $A=16 \mathrm{deg}^{2}$ and the galaxy intrinsic ellipticity rms to 0.3 . Figure 7 shows the confidence regions, assuming weak priors on pairs of hidden parameters. From the four parameters to be determined, we can construct 6 pairs of parameters, and for each pair, the other two parameters are marginalized, in such a way that $\Omega_{\mathrm{m}} \in[0.2,0.4], \sigma_{8} \in[0.8,1.1], \Gamma \in[0.1,0.3]$ and $z_{\mathrm{s}} \in[0.4,0.5]$. The marginalization intervals are chosen to be consistent with realistic constraints coming from other experiments, especially from the Cosmic Microwave Background (e.g., Sievers et al. 2002), the $2 \mathrm{dF}$ (Lahav et al. 2002) and the SLOAN (Szalay et al. 2001) results, and photometric redshifts. Note that we always fix the cosmological constant to $\Omega_{\Lambda}=1-\Omega_{\mathrm{m}}$, that is we assume the (correct) flat geometry. The strongest constraints are found for the $\Omega_{\mathrm{m}}-\sigma_{8}, \Gamma-\sigma_{8}$ and $\Omega_{\mathrm{m}}-\Gamma$ pairs, suggesting that once the redshift distribution of the sources is known we can obtain stringent constraints on the cosmological parameters from cosmic shear. The degeneracy directions can be better studied with a strong prior likelihood analysis as shown in Fig. 8. On this plot, we assume that the two hidden parameters of each constrained pair are known and fixed at their true value. Again we see that the best constraints come from pairs of parameters which exclude the redshift information. It is remarkable that the degeneracy among these parameters can be broken quite efficiently, given that the cosmic shear signal has so few "spectral features", compared to the cosmic microwave background for instance. In fact, the degeneracy can be broken when using linear and non-linear scales simultaneously (as discussed in 


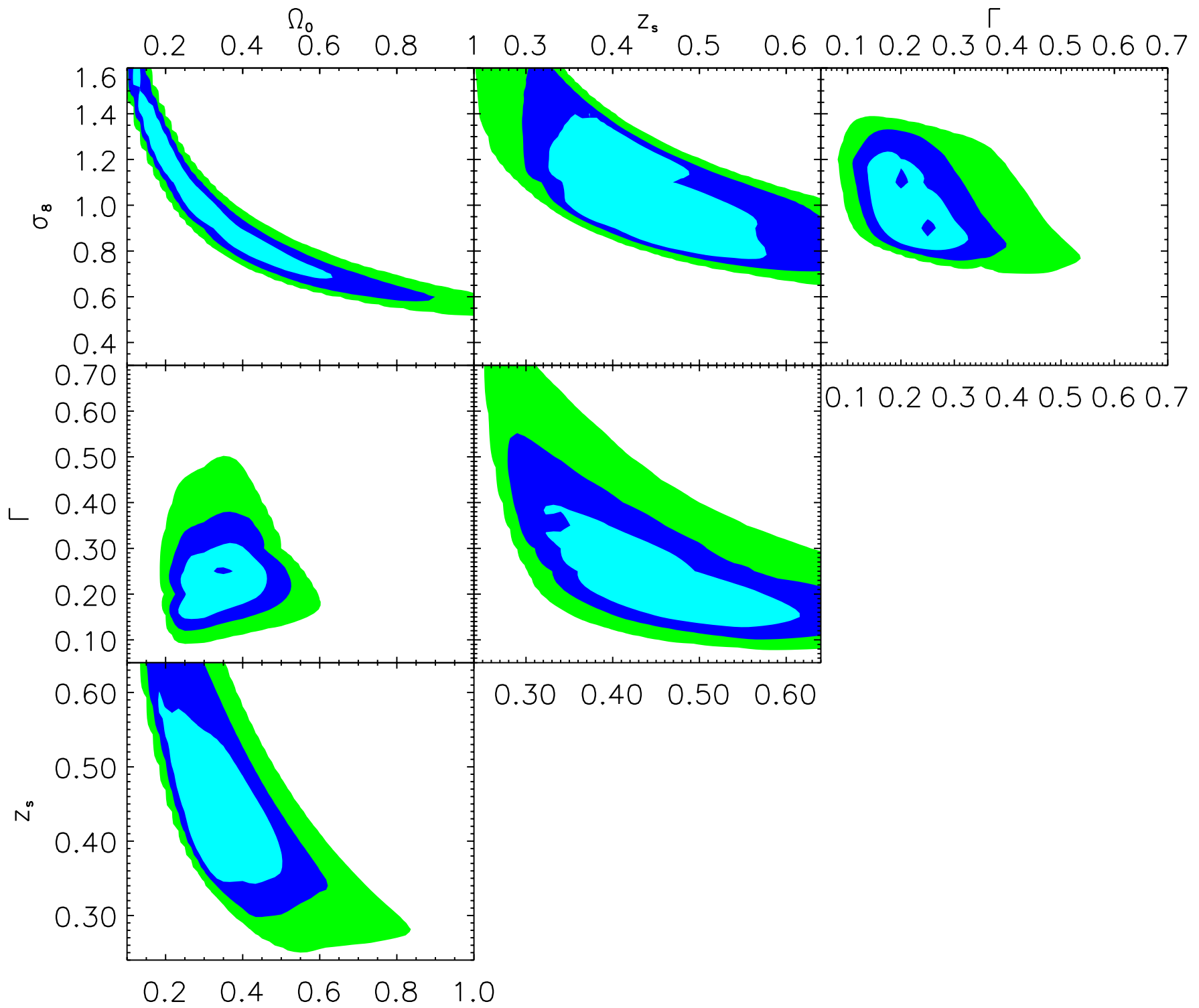

Fig. 7. 1- $\sigma, 2-\sigma$ and 3- $\sigma$ confidence contours for the maximum likelihood analysis on the four parameters $\Omega_{\mathrm{m}}, \sigma_{8}, \Gamma$ and the source redshift parameter $z_{\mathrm{s}}$ (see text). The six possible pairs of parameters are displayed. On each figure, the two hidden parameters are marginalized such that $\Omega_{\mathrm{m}} \in[0.2,0.4], \sigma_{8} \in[0.8,1.1], \Gamma \in[0.1,0.3]$ and $z_{\mathrm{s}} \in[0.4,0.5]$, and the cosmological constant is fixed to $\Omega_{\Lambda}=1-\Omega_{\mathrm{m}}$. The reference model is $\Omega_{\mathrm{m}}=0.3, \sigma_{8}=1, \Gamma=0.21$ and $z_{\mathrm{s}}=0.44$. The survey area is $A=16 \mathrm{deg}^{2}$, the galaxy ellipticity rms is 0.3 , and the correlation functions are measured in the range $00^{\prime} 6 \vartheta \leq 30^{\prime}$.

Jain \& Seljak 1997). One would obtain an even better degeneracy breaking by going further into the linear regime (up to 1 or 2 degrees). Also, it is interesting to note that the parameters which are least affected by the marginalization (compare Figs. 7 and 8 ) is the $\Omega_{\mathrm{m}}-\sigma_{8}$ pair, which is most directly comparable to cluster normalization constraints (see a discussion on the comparison between cluster normalization and cosmic shear constraints in van Waerbeke et al. 2002).

\section{Conclusions}

In this paper we have obtained general expressions for the covariance of an estimator for the shear correlation function as it is determined from cosmic shear data. Using the approximation that the four-point function of the shear separates in products of two-point functions, the covariance can be expressed directly in terms of the correlation functions, as given in (23)-(25) and can, for a given data set, be calculated directly. The covariance of the correlation functions depends on the number of pairs that enter their estimate, which in turn depends on the solid angle covered by the survey and the survey geometry (see also Kaiser 1998); in addition, it depends on the intrinsic galaxy ellipticities and the number density of galaxies.

Next, considering a survey geometry of a single compact region of solid angle $A$, we have calculated the ensemble average of the covariances, using approximations which a valid for separations $\ll \sqrt{A}$. The ensemble average of the covariances can then be 


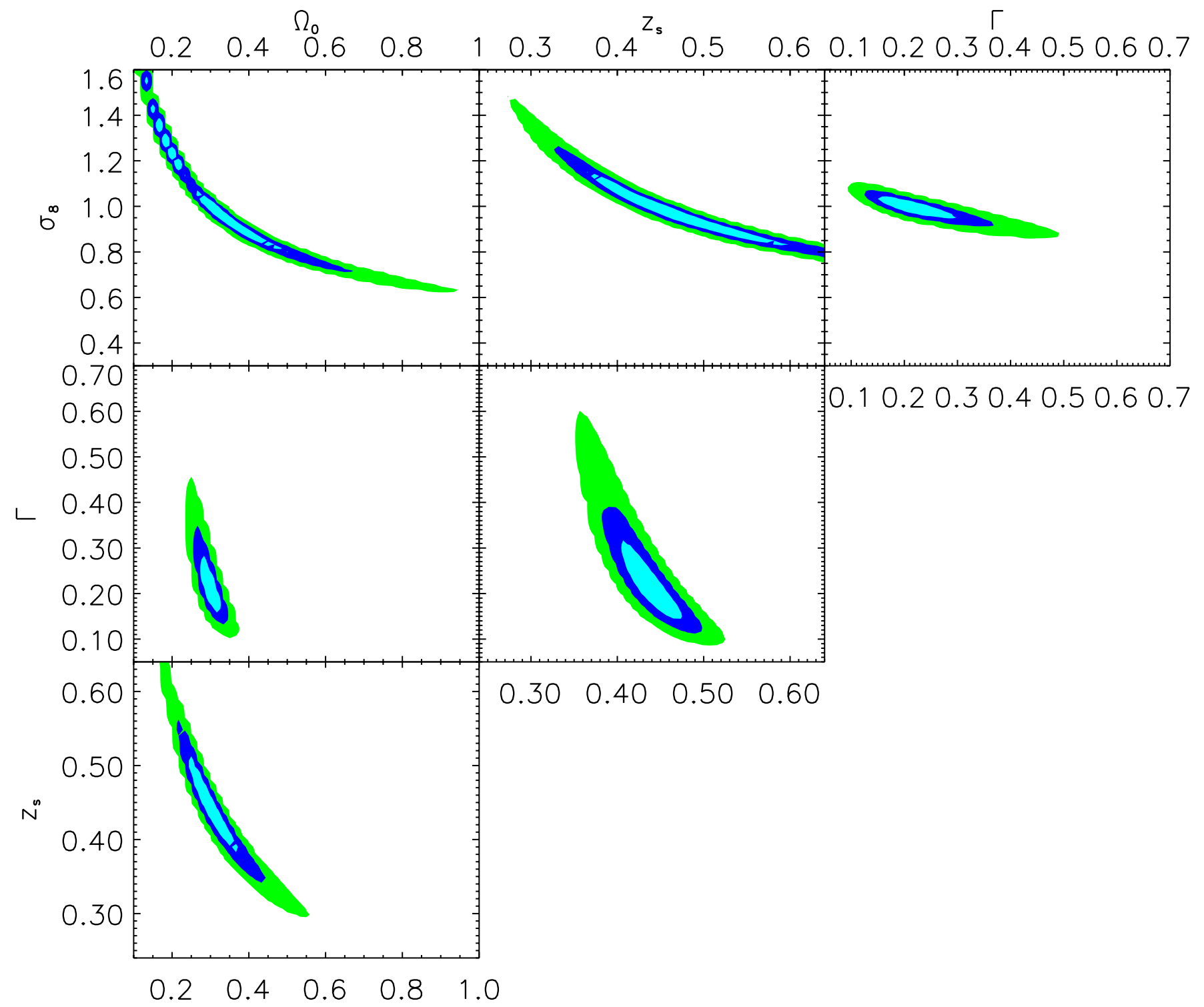

Fig. 8. Same as Fig. 7 with strong priors: in each figure, the two hidden parameters as assumed to be known perfectly. These plots show the degeneracy directions among all the possible pairs of parameters obtained from $\Omega_{\mathrm{m}}, \sigma_{8}, \Gamma$ and $z_{\mathrm{s}}$. Note that the wiggles at the edge of the contours are not real features of the probability constraints. Their are inherent to the sampling limitation of the 4-dimensional cube of models given the memory limit of our machines. Also note that the upper left panel is the analogue of Fig. 6, but without the scaling employed there.

reduced to integrals which are readily evaluated numerically. The estimate for the correlation function $\xi_{-}(\vartheta)$ decorrelates quickly,

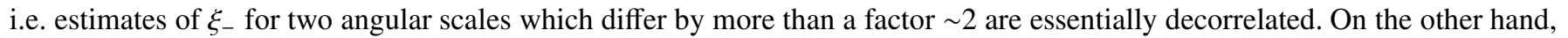
the estimates of $\xi_{+}$are correlated over much larger angular scales. The cross-correlation between $\xi_{+}\left(\vartheta_{1}\right)$ and $\xi_{-}\left(\vartheta_{2}\right)$ is significant for $\vartheta_{1} \lesssim \vartheta_{2}$, which is due to the properties of the different filters with which these correlation functions are related to the power spectrum $P_{\kappa}(\ell)$.

Using these ensemble-averaged covariances for the correlation functions, we have obtained the covariances for other secondorder measures of the cosmic shear, primarily the aperture mass dispersion and the power spectrum. Of particular interest is the reconstruction of the power spectrum $P_{K}(\ell)$ from the correlation functions; we have constructed a simple estimator for $P_{K}$ and the band powers of it in terms of the $\xi$ 's and found that the band power can be obtained with surprisingly large accuracy from even a moderately-sized cosmic shear survey. Finally, we have investigated the confidence regions for the most relevant cosmological parameters $\left(\Omega_{\mathrm{m}}, \sigma_{8}, \Gamma\right.$ and $\left.z_{\mathrm{s}}\right)$ with a maximum likelihood approach. We studied our ability to constrain simultaneously these parameters from a measurement of the shear correlation function, as well as the effect of some level of lack of knowledge using the marginalization technique.

In a future paper, we shall investigate strategies for conducting cosmic shear surveys by optimizing the survey geometry. 
Acknowledgements. We thank the referee Dipak Munshi for his constructive comments on the manuscript. This work was supported by the TMR Network "Gravitational Lensing: New Constraints on Cosmology and the Distribution of Dark Matter" of the EC under contract No. ERBFMRX-CT97-0172, by the German Ministry for Science and Education (BMBF) through the DLR under the project 50 OR 0106, and by the Deutsche Forschungsgemeinschaft under the project SCHN 342/3-1.

\section{References}

Bacon, D. J., Refregier, A. R., \& Ellis, R. S. 2000, MNRAS, 318, 625

Bacon, D. J., Massey, R., Refregier, A. R., \& Ellis, R. S. 2002, MNRAS, submitted [astro-ph/0203134]

Bardeen, J. M., Bond, J. R., Kaiser, N., \& Szalay, A. S. 1986, ApJ, 304, 15

Bartelmann, M., \& Schneider, P. 1999, A\&A, 345, 17

Bartelmann, M., \& Schneider, P. 2001, Phys. Rep., 340, 291

Benitez, N., Sanz, J. L., \& Martinez-Gonzalez, E. 2001, MNRAS, 320, 241

Bernardeau, F., van Waerbeke, L., \& Mellier, Y. 1997, A\&A, 322, 1

Bernardeau, F., van Waerbeke, L., \& Mellier, Y. 2002a [astro-ph/0201029]

Bernardeau, F., Mellier, Y., \& van Waerbeke, L. 2002b, A\&A, 389, L28

Blandford, R. D., Saust, A. B., Brainerd, T. G., \& Villumsen, J. V. 1991, MNRAS, 251, 600

Brown, M., Taylor, A., Hambly, N., \& Dye, S. 2002, MNRAS, 333, 501

Catelan, P., Kamionkowski, M., \& Blandford, R. D. 2001, MNRAS, 320, L7

Crittenden, R. G., Natarajan, P., Pen, U.-L., \& Theuns, T. 2002, ApJ, 568, 20 (C02)

Croft, R. A. C., \& Metzler, C. A. 2001, ApJ, 545, 561

Dolag, K., \& Bartelmann, M. 1997, MNRAS, 291, 446

Erben, T., Van Waerbeke, L., Bertin, E., Mellier, Y., \& Schneider, P. 2001, A\&A, 366, 717

Hämmerle, H., Miralles, J.-M., Schneider, P., et al. 2002, A\&A, 385, 743

Hamilton, A. J. S., Matthews, A., Kumar, P., \& Lu, E. 1991, ApJ, 374, L1

Heavens, A. F., Refregier, A., \& Heymans, C. E. C. 2000, MNRAS, 319, 649

Hoekstra, H., Franx, M., \& Kuijken, K. 2000, ApJ, 532, 88

Hoekstra, H., Yee, H., \& Gladders, M., ApJ, in press [astro-ph/0204295]

Hu, W., \& White, M. 2001, ApJ, 554, 67

Jain, B., \& Seljak, U. 1997, ApJ, 484, 560

Jain, B., Seljak, U., \& White, S. D. M. 2000, ApJ, 530, 547

Kaiser, N. 1992, ApJ, 388, 272

Kaiser, N. 1998, ApJ, 498, 26

Kaiser, N., Wilson, G., \& Luppino, G. 2000 [astro-ph/0003338]

Lahav, O., Bridle, S. L., Percival, W. J., et al. 2002, MNRAS, 333, 961

Mackey, J., White, M., \& Kamionkowski, M. 2002, MNRAS, 332, 788

Maoli, R., van Waerbee, L., Mellier, Y., et al. 2001, A\&A, 368, 766

Mellier, Y. 1999, ARA\&A, 37, 127

Menard, B., \& Bartelmann, M. 2002, A\&A, 386, 784

Miralda-Escudé, J. 1991, ApJ, 380, 1

Munshi, D., \& Coles, P. 2000 [astro-ph/0003481]

Munshi, D., \& Jain, B. 2001, MNRAS, 322, 107

Peacock, J. A., \& Dodds, S. J. 1996, MNRAS, 280, L19

Pen, U.-L., Jounghun, L., \& Seljak, U. 2000, ApJ, 543, 107

Pen, U.-L., van Waerbeke, L., \& Mellier, Y. 2002, ApJ, 567, 31

Refregier, A., Rhodes, J., \& Groth, E. J. 2002, ApJ, 572, 131

Rhodes, J., Refregier, A., \& Groth, E. J. 2001, ApJ, 552, L85

Schneider, P. 1996, MNRAS, 283, 837

Schneider, P., van Waerbeke, L., Jain, B., \& Kruse, G. 1998, MNRAS, 296, 873 (SvWJK)

Schneider, P., van Waerbeke, L., \& Mellier, Y. 2002, A\&A, 389, 729 (SvWM)

Seljak, U. 1998, ApJ, 506, 64

Sievers, J. L., Bond, J. R., Cartwright, J. K., et al. 2002, ApJ, submitted [astro-ph/0205387]

Smith, R. E., Peacock, J. A., Jenkins, A., et al. 2002 [astro-ph/0207664]

Szalay, A. S., Jain, B., Matsubara, T., et al. 2001, ApJ, submitted [astro-ph/0107419]

van Waerbeke, L. 2000, MNRAS, 313, 524

van Waerbeke, L., Bernardeau, F., \& Mellier, Y. 1999, A\&A, 243, 15

van Waerbeke, L., Mellier, Y., Erben, T., et al. 2000, A\&A, 358, 30

van Waerbeke, L., Mellier, Y., Radovich, M., et al. 2001, A\&A, 374, 757

van Waerbeke, L., Mellier, Y., Pello, R., et al. 2002 [astro-ph/0202503]

White, M., \& Hu, W. 2000, ApJ, 537, 1

Wittman, D. M., Tyson, J. A., Kirkman, D., Dell'Antonio, I., \& Bernstein, G. 2000, Nature, 405, 143 